\title{
Embedded waveshifting fiber readout of long scintillators
}

\author{
R. Wojcik *, B. Kross, S. Majewski, A.G. Weisenberger, C. Zorn \\ Detector Group, Continuous Electron Beam Accelerator Facility, 12000 Jefferson Avenue, Newport News, VA 23606, USA
}

(Received 8 October 1993)

\begin{abstract}
We have tested a method of reading out light from long scintillators by embedding waveshifting fibers in longitudinal grooves created in the scintillators which are then connected to a photomultiplier (PMT). With PMTs on both ends of the fibers we have obtained attenuation lengths of over $5 \mathrm{~m}$ and light outputs of over 5 photoelectrons per MeV from both ends of a $4 \mathrm{~m}$ long BC408 scintillator with five embedded $2 \mathrm{~mm}$ diameter BCF92 fast green waveshifting fibers using a Hamamatsu green extended R580-17 PMT. With a reflector at one end of the fibers, we have easily obtained over $10 \mathrm{~m}$ attenuation lengths. Also presented are results using inexpensive acrylic scintillators with this method and measurements of a new experimental scintillator from Bicron with over a $5 \mathrm{~m}$ attenuation length.
\end{abstract}

\section{Introduction}

Calorimetric detectors based on scintillating fibers [1] or waveshifting fibers [2,3] are popular today and there is an active research going on to improve their properties such as light yield, uniformity of response, timing and radiation resistance. For scintillator tiles, wavelength shifting fibers have been used in either a "shish-kebab" (or "shashlik") configuration with fibers perpendicular to the tiles [2], or with fibers placed in the scintillator plane either by embedding them in grooves [3] or simply placing them at the scintillator edges [3a]. In sandwich designs that use long scintillators when, due to space limitations or a requirement of an economical readout, photomultipliers (PMTs) can not be placed at the end(s) of the scintillators, light is collected and channeled to a PMT through some sort of light guide. In the past, different designs have been tried. One is a waveshifting bar coupled to the ends of the scintillator stack. However this design suffers from a relatively low light output. Another is a light guide made of clear fibers coupled to the end of the scintillators [4]. To get enough light out to detect efficiently low energy particles and neutrons, one must use for example 20 or more $3 \mathrm{~mm}$ fibers which makes fabrication of these light guides rather tedious. The attenuation length for both these methods is also limited by the attenuation length of the scintillator which is heavily dependent on the bulk material and surface quality of the scintillator. By embedding wave shifting fibers in

* Corresponding author. longitudinal grooves in the scintillator, one is able to obtain light outputs comparable with the fiber light guide with easier fabrication while greatly increasing the attenuation length which now depends on the attenuation length of the fibers. Shifting the light to longer wavelengths should also make the embeddedfiber detector much more radiation hard.

\section{Experimental setup}

Except where noted, all samples in our tests had cross sections of $1 \mathrm{~cm} \times 10 \mathrm{~cm}$ with five 2 -mm-wide and 2-mm-deep grooves machined longitudinally in them with a specially prepared tool. The sample length and fiber distance to the PMT varied depending on the test. All fibers were $2 \mathrm{~mm}$ in diameter and were prepared by sanding their ends flat and then polishing with $1 \mu \mathrm{m}$ diamond paste polishing compound [5]. Two-cm-long regions $3 \mathrm{~cm}$ from the PMT end were painted black to eliminate cladding light and the opposite ends were usually painted black to act as a light dump. The PMT at the fiber end was calibrated by scattering a very small amount of light into the fibers from a pulsed light emitting diode so that there was much less than one photo-electron (PE) detected on average in order to obtain a single PE calibration spectrum. This enabled absolute calibration of the detected signal in photoelectrons. All absolute numbers of photoelectrons are good to $\leq 10 \%$. The PMT used was the Hamamatsu R580-17 green extended bialkali PMT. The $1 \mathrm{MeV}$ measurements were made by gating 
Table 1

Light output for $1 \mathrm{MeV}$ energy deposited and for three fiber mounting methods obtained with a $16 \mathrm{~cm}$ long BC408 scintillator wrapped in double-sided 1 mil aluminized Mylar with 2.5 $\mathrm{m}$ long BCF92 fibers

\begin{tabular}{llll}
\hline & Glued & Non-glued & Side-mount \\
\hline Number of photoelectrons & 12 & 6.5 & 4.5 \\
\hline
\end{tabular}

on the scintillation produced in the scintillator from the $1 \mathrm{MeV}$ conversion electron of a ${ }^{207} \mathrm{Bi}$ source as seen by a second PMT looking through a small hole in the wrapping material near the source.

\section{Comparative tests}

\subsection{Coupling methods}

We tested three different methods to couple fibers to the scintillator - laid in the grooves without glue, glued in the grooves, and side-mounted on the edge surface. Fig. 1a shows a picture and a sketch of a grooved scintillator with five wave shifting fibers laid in the grooves. The side-mounted technique does not require grooves in the scintillator and is shown in Fig. 1b. Table 1 shows the results of the different methods obtained with a $16 \mathrm{~cm}$ long BC408 scintillator wrapped in double-sided 1 mil aluminized Mylar [6] and with 2.5 $\mathrm{m}$ long BCF92 fibers. The scintillator was placed close to the far end of the fibers and $1.9 \mathrm{~m}$ from the PMT. The fibers placed in the grooves without glue had about half the light output as fibers glued in with BC600 optical glue [7]; however, gluing is a delicate and time consuming process. The main drawback to the non-glued and side-mounted fibers is that the scintillator has to be tightly wrapped to hold the fibers in place. The side-mounted fibers had about $70 \%$ the light output of the non-glued fibers but required no machining of the plate scintillator.

\subsection{Wrapping materials}

We also tested the effect of different wrapping materials on the light output. Fig. 2 shows the results
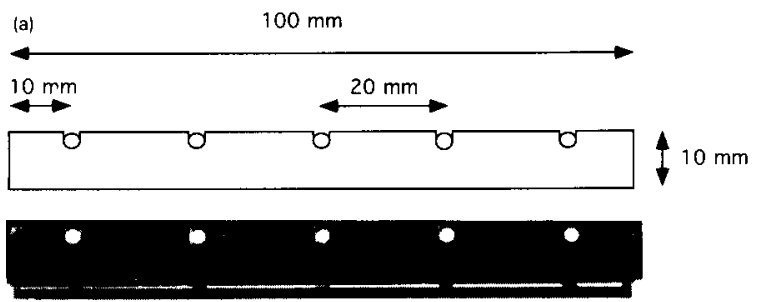

Fig. 1. A picture and a sketch of a grooved scintillator with five waveshifting fibers: (a) laid in the grooves, (b) the side-mounted

technique does not require grooves in the scintillator.

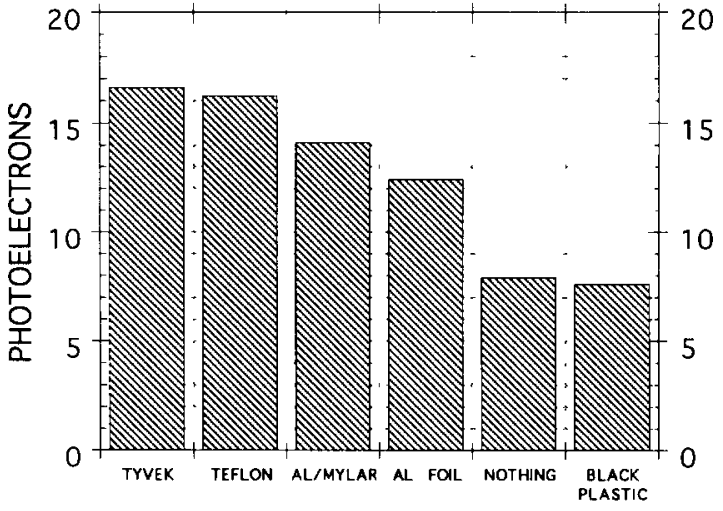

F1g. 2. The effect of different wrapping materials on the light output from a $20 \mathrm{~cm}$ long $\mathrm{BC} 408$ scintillator with BCF91A green waveshifting fibers glued into the grooves

for a $20 \mathrm{~cm}$ long BC408 scintillator $46 \mathrm{~cm}$ from the PMT with $110 \mathrm{~cm}$ long BCF91A green waveshifting fibers glued into the grooves. The tests were made by triggering on the $1 \mathrm{MeV}$ conversion electron of ${ }^{207} \mathrm{Bi}$. Tyvek white paper [8,9] gives the highest light output; however the uniformity is not as good as aluminized Mylar due to local variations in material thickness and reflectivity (Fig. 3). Some recent communicated information, however, suggests that a new style of Tyvek (type 55D), or the use of PET paper, may produce a superior uniformity [10]. The curves in Fig. 3 were obtained by measuring PMT current when scanning a 4 $m$ long $\mathrm{BC} 408$ scintillator with an beta electron beam from a strong ${ }^{106} \mathrm{Ru}$ source. Tests were also made to see if there would be any change in the optical properties due to the wrapping material being pressed against the scintillator. The light output of a two-meter long BC404 scintillator with $2.5 \mathrm{~m}$ long BCF92 fibers wrapped in either black paper or aluminized Mylar was measured before and after the application of a pressure of about 1 psi $\left(70 \mathrm{~g} / \mathrm{cm}^{2}\right)$. No change was seen in the light output after two days. The only wrapping material reported to change its optical characteristics under pressure is Teflon [10], however there have been anecdotal reports concerning the adhesion of aluminum from aluminized Mylar to surfaces of materials such as lead glass.

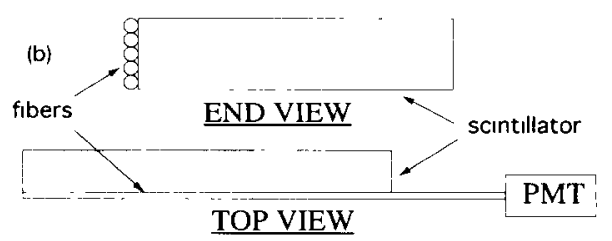




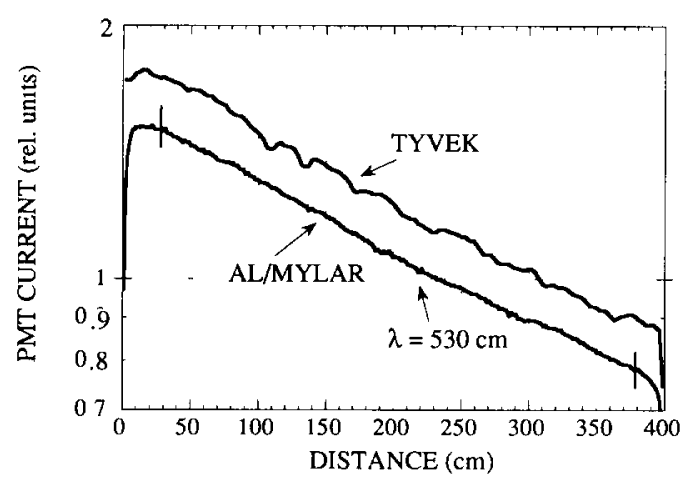

Fig. 3. Light output versus the position of a collimated beam of beta electrons from a ${ }^{106} \mathrm{Ru}$ source for two wrapping materials: Tyvek and aluminized Mylar.

\subsection{Response uniformity}

Both transverse and longitudinal uniformity of response were studied for non-traversing particles. The transverse uniformity of response depends on the spacing of the grooves and the number of fibers. For the uniform spacing of $16.7 \mathrm{~mm}$ and five fibers, the signal decreased by $6.5 \%$ towards the edges when tested with a 5-mm-wide beam from an $8-\mathrm{keV} \mathrm{X}$-ray generator irradiating the side opposite the grooves (Fig. 4a). Our best results came from spacing the grooves $1 \mathrm{~cm}$ from each edge and $2 \mathrm{~cm}$ apart (as in Fig. 1a), which gave less than a $2 \%$ variation. Fig. $4 \mathrm{~b}$ shows the results of scanning with an X-ray beam across the center of a $1 \mathrm{~m}$ long BC408 scintillator with $2.5 \mathrm{~m}$ long BCF92 nonglued fibers and $1 \mathrm{~m}$ from the PMT.

The side-mounted five fibers show about a $6 \%$ variation (Fig. 5). As others have found [10], some light is also produced by the $\mathrm{X}$-rays in the waveshifting fibers, however it was found to be a very small amount that does not affect the results. It was also seen in these measurements that the light is shared approxi-

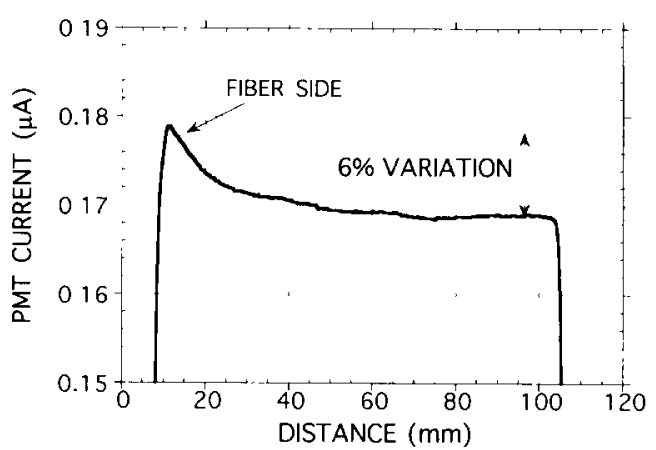

Fig. 5. Cross uniformity scan for five side-mounted fibers (as schematically shown in Fig. 1b).

mately equally among the fibers. This is shown in the lower curves of Figs. 4a and 4b where, with only three fibers connected to the PMT (but with all five fibers in place), the signal dropped to about $60 \%$ of the light detected from all five fibers. Attenuation measurements, where we also tried reading out the fibers individually (Fig. 6), show that this equal sharing of light tends to have an averaging effect on the total light output. If one fiber does not collect the light due to scattering from the imperfections at the groove or fiber surface, other fibers compensate by collecting that scattered light so that the total light output does not show these variations.

The longitudinal signal uniformity was measured with an X-ray beam using a $4 \mathrm{~m} \mathrm{BC} 408$ scintillator with $6 \mathrm{~m} \mathrm{BCF} 92$ fibers (Fig. 7). Based on the experience with wrapping materials (Fig. 2), the scintillator was wrapped with aluminized Mylar. The summed output of the left and right PMTs reading out the ends of the fibers showed less than a $10 \%$ variation over the length of the scintillator and local fluctuations of under $2 \%$ demonstrating very good uniformity in light conversion and transport in the fibers with an attenuation length on the order of $5.5 \mathrm{~m}$ in both outputs.
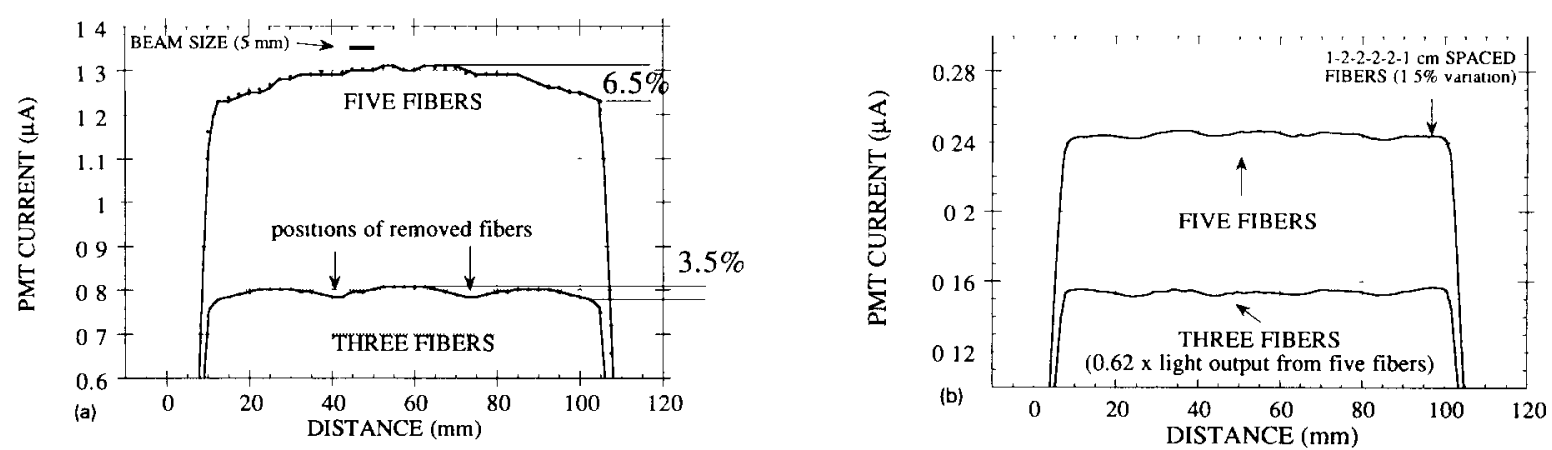

Fig. 4. The transverse response uniformity for two spacings of the grooves tested with a 5-mm-wide 8 keV X-ray beam: (a) uniform spacing of $16.7 \mathrm{~mm}$, and (b) optimized spacing of the grooves: $1 \mathrm{~cm}$ from each edge and $2 \mathrm{~cm}$ apart (as in Fig. 1a). 


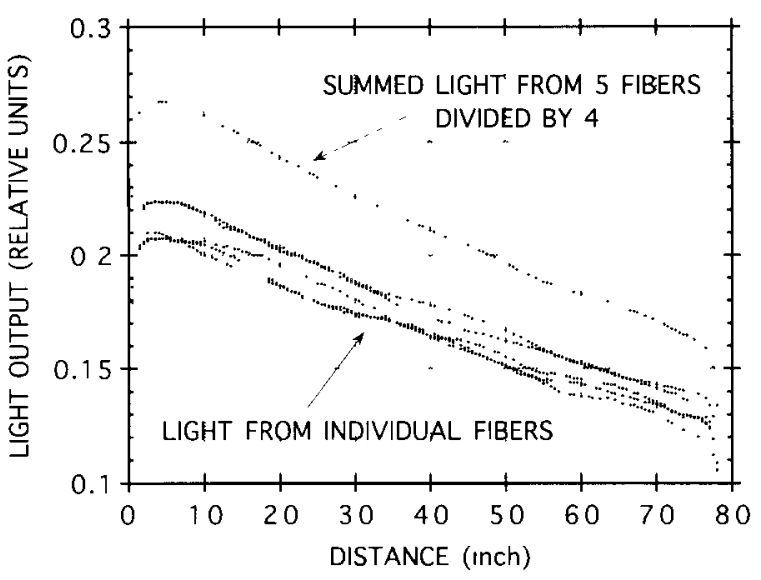

Fig. 6. Individual and summed fiber readout for attenuation scans of a BC408-BCF92 scintillator-fiber combination.

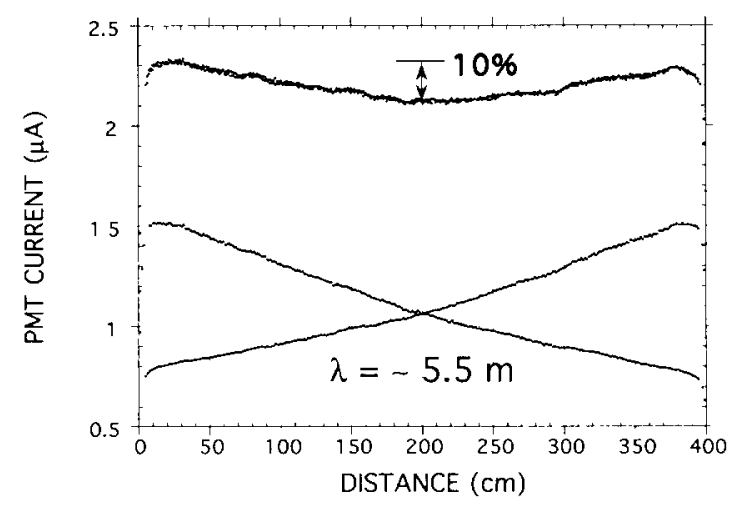

Fig. 7. Longitudinal signal uniformity measured with an X-ray

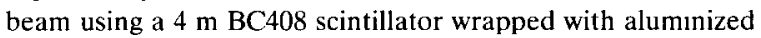
Mylar and $6 \mathrm{~m}$ long BCF92 fibers. The summed output of the left and right PMTs is also shown.

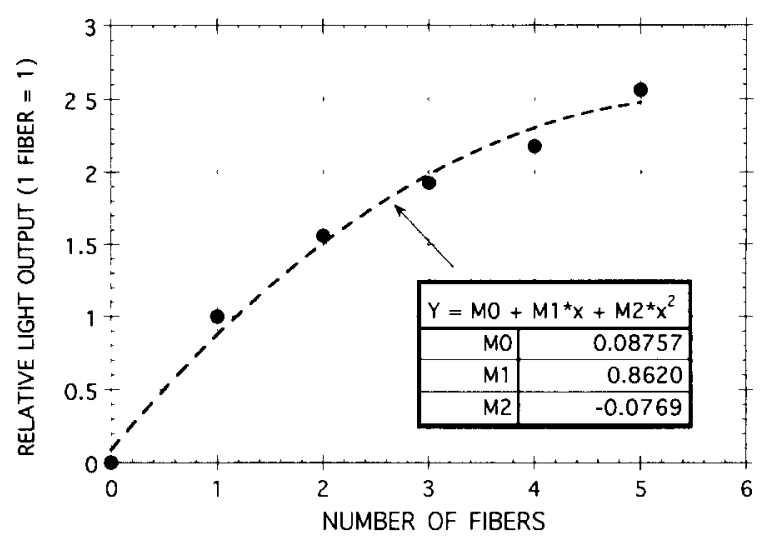

Fig. 9. Relative light output for aluminized Mylar wrapping versus number of fibers.

\subsection{Light output versus number of grooves}

Tests were also made to see the effect different numbers of grooves had on the light output and transverse uniformity of the scintillator. We used a set of five $19.5 \mathrm{~cm}$ long BC414 scintillators wrapped with aluminized Mylar with increasing numbers of grooves, from one to five, along with the corresponding number of $2.5 \mathrm{~m}$ long BCF92 fibers. The scintillators were scanned transversally using an X-ray beam, as described before, with a $50 \mathrm{~cm}$ scintillator-to-PMT distance (Fig. 8a). Even with only one readout fiber, the transverse non-uniformity was still only $10 \%$. The non-uniformity increases (and signal decreases) for black paper wrapped scintillators (Fig. 8b). In the case of aluminized Mylar going from one groove to five grooves increases the light yield by 2.5 times and shows the saturation in response versus number of fibers a-posteriori confirming the correctness of our choice to use five grooves (Fig. 9).
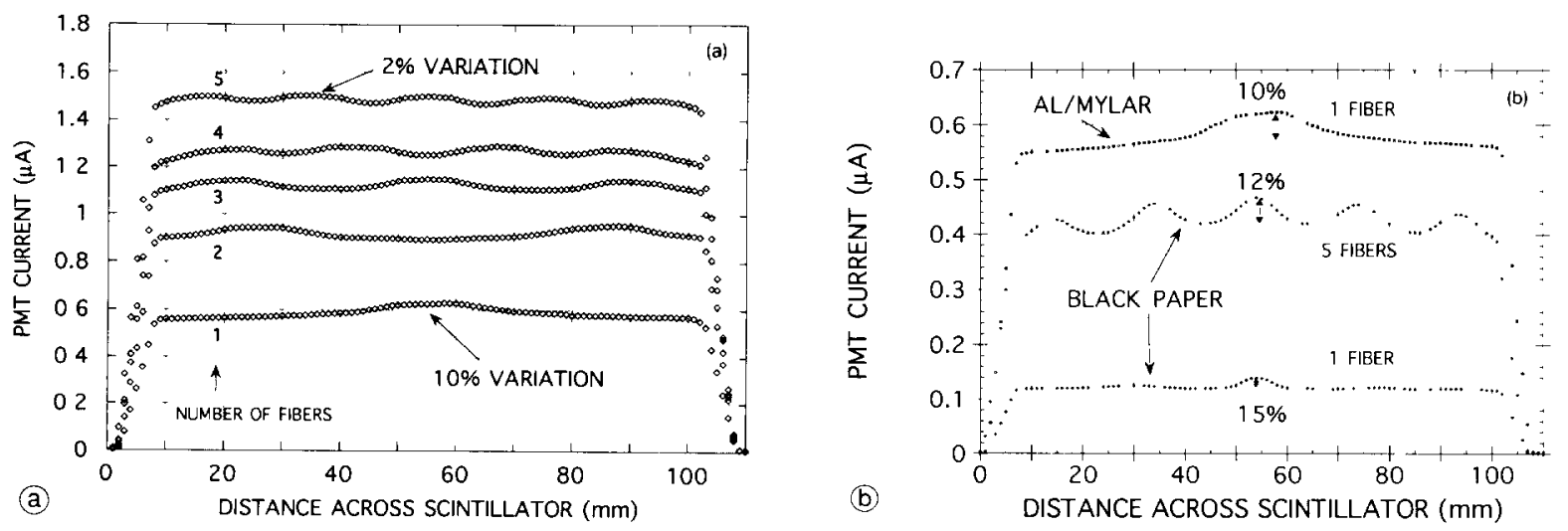

Fig. 8. Transverse scans using an X-ray beam for one to five readout fibers for aluminized Mylar (a) and black paper wrapping (b). 

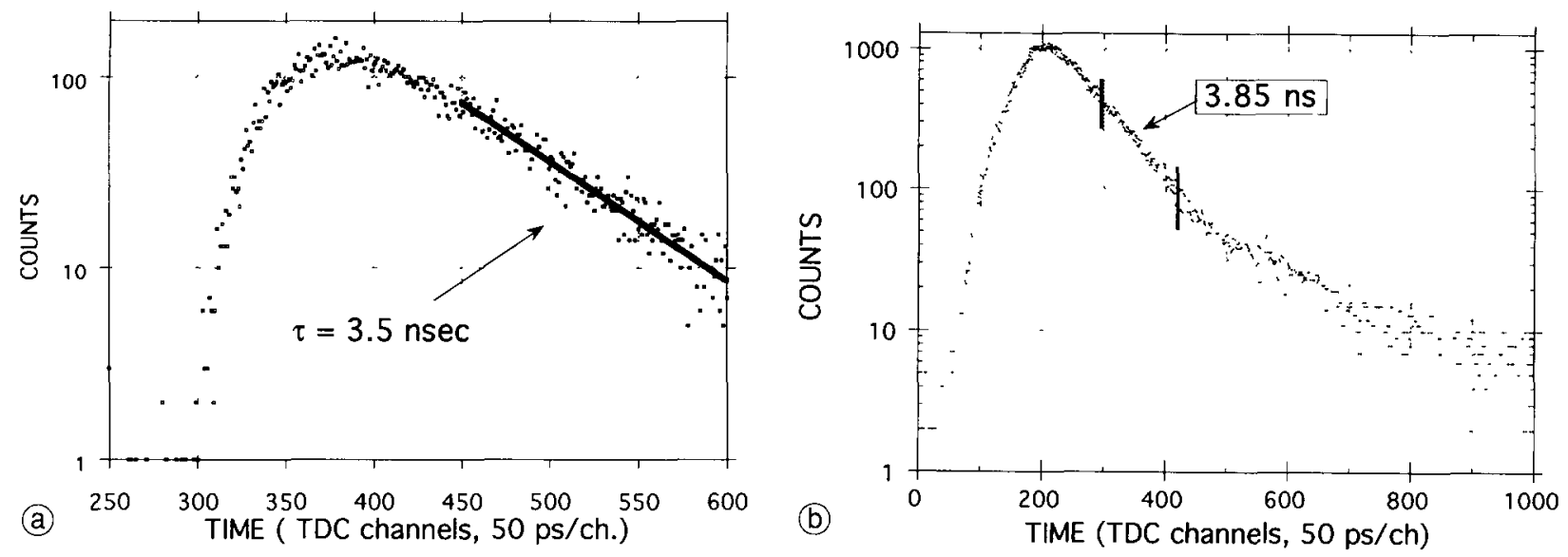

Fig. 10. The decay constant of the BC408-BCF92 scintillator-fiber combination measured with a single photon technique with electrons from ${ }^{207} \mathrm{Bi}$ incident on a $2 \mathrm{~cm}$ long grooved $\mathrm{BC} 408$ scintillator (a) and with a Teflon-wrapped $8 \mathrm{~cm}$ long BC408 scintillator of a $2 \mathrm{~cm} \times 1 \mathrm{~cm}$ cross section (b).

One has to emphasize that the uniformity of response in the case of penetrating particles will be different from the results obtained in our laboratory tests for two reasons. First, light generation will be distributed differently in depth, especially as compared to the case of the X-ray beam tests with X-rays stopping in the first millimeters of the scintillator material. Secondly, the grooving leads to the removal of the active material. Proper simulations should be performed for a particular application to define if this poses a problem. In the shower detector application, the transversal size of the grooves is small as compared to the transversal shower size and the grooves can be spread out differently in the different sampling layers to avoid accumulation of lower energy losses in the same positions of the scintillator stack. If this is still not acceptable, the waveshifting fibers can be made slightly scintillating to compensate for most of the removed material. Also, with the new progress with double clad fibers (see section 9) $1 \mathrm{~mm}$ fibers can be used instead of $2 \mathrm{~mm}$ fibers, allowing for a decrease in the size of the grooves.

\subsection{Timing and photo-statistics studies}

We were interested in the fastest possible readout and found that the only choice for fast green waveshifting fibers is BCF92 from Bicron. The decay constant of the BC408-BCF92 scintillator-fiber combination was measured in a single photon mode to confirm that BCF92 is a fast wavelength shifter with a decay time of $2.8 \mathrm{~ns}$ [11] and to evaluate the effect of the embeddedfiber technique on the timing properties. We used electrons from ${ }^{207} \mathrm{Bi}$ incident on a $2 \mathrm{~cm}$ long grooved BC408 scintillator with a decay time of $2.1 \mathrm{~ns}$ [11] to excite the fibers. A second PMT coupled directly to the scintillator produced the start pulses and the light from one of the waveshifting fibers passing through a pinhole produced the stop pulses. Our finding of a $3.5 \mathrm{~ns}$ decay time (Fig. 10a) confirms that BCF92 is indeed a fast wavelength shifter and shows that the embeddedfiber technique, when using BCF92, does not greatly change the decay time compared to that measured directly from the scintillator. In another measurement, for a Teflon-wrapped $8 \mathrm{~cm}$ long BC408 scintillator of a $2 \mathrm{~cm} \times 1 \mathrm{~cm}$ cross section with five BCF92 fibers, we obtained a similar result of 3.85 ns for the fast decay component (Fig. 10b). In a long scintillator, there is some worsening of the decay time as is seen in Fig. 11 for a $2 \mathrm{~m}$ long BC414 scintillator, which has a $1.8 \mathrm{~ns}$ decay time [11]. Using a ${ }^{207} \mathrm{Bi}$ source $50 \mathrm{~cm}$ from the PMT end of the scintillator with waveshifting fibers extending $50 \mathrm{~cm}$ beyond the scintillator edge the decay

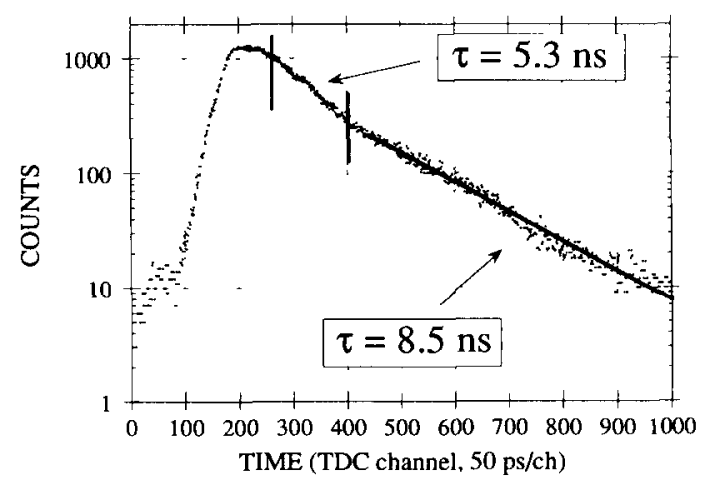

Fig. 11. The decay constant of the BC414-BCF92 scintillatorfiber combination measured in a $2 \mathrm{~m}$ long $\mathrm{BC} 414$ scintillator wrapped with aluminized Mylar. Far fiber ends and both scintillator ends were painted with black paint to prevent back reflection of light. 


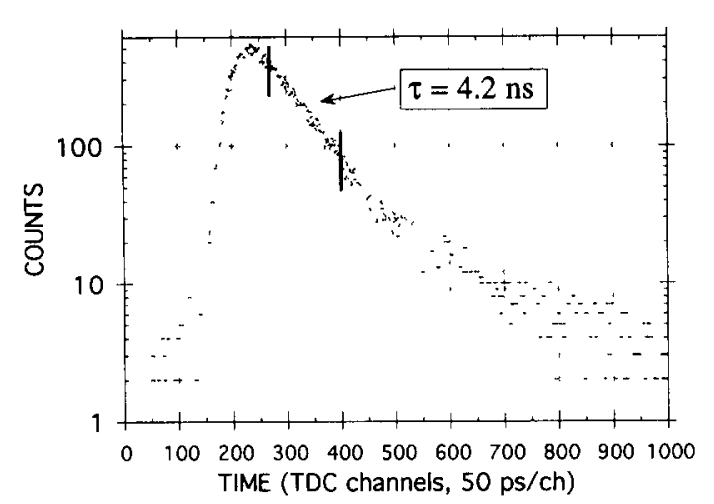

Fig. 12. The single photoelectron fast decay time measured for a $3 \mathrm{~m}$ long $\mathrm{BC} 412$ scintillator with the ${ }^{207} \mathrm{Bi}$ source placed $50 \mathrm{~cm}$ from the PMT end of the scintillator using the clear fiber light guide attached to the PMT end.

time was found to be 5.3 ns. The scintillator was wrapped with aluminized Mylar but the far fiber ends and both scintillator ends were painted with black paint to prevent the reflection of light. To compare with the direct readout method, a single photoelectron fast decay time of $4.2 \mathrm{~ns}$ was measured for a $3 \mathrm{~m}$ long BC412 scintillator, which has a 3.3 ns decay constant, with the ${ }^{207} \mathrm{Bi}$ source placed $50 \mathrm{~cm}$ from the PMT end of the scintillator and with the clear fiber light guide attached at the PMT end (Fig. 12). The clear fiber light guide was made of twenty $50 \mathrm{~cm}$ long and $3 \mathrm{~mm}$ diameter acrylic clad polystyrene fibers. This timing difference, however, does not significantly harm the timing resolution obtainable with waveshifting fibers, and due to factors discussed below can actually, in some cases, be better with fibers.

The main limitation to timing resolution, we found, was photon statistics for both the waveshifting fiber and clear fiber light guide readout. Because of low light levels there is a statistical time jitter in the arrival of the first photoelectrons which produces a timing resolution on the order of $3.25 \mathrm{~ns}$ fwhm for $1 \mathrm{MeV}$ ${ }^{207} \mathrm{Bi}$ electrons in a $2 \mathrm{~m}$ long $\mathrm{BC} 414$ scintillator with BCF92 fibers (Fig. 13) for an average of 8.7 photoelectrons detected (Fig. 14). This was the same scintillator piece for which the single photoelectron curve from Fig. 11 was obtained. Leading edge timing with the threshold set well below the average SPE pulse amplitude was used.

In the several measurements performed with cosmic rays we have noticed that, in every case, the obtained time resolution is worse than the one extrapolated from the $1 \mathrm{MeV}$ energy loss from ${ }^{207} \mathrm{Bi}$ electrons. We speculate that this is related to the differences in the light generation geometry with the energy deposition from ${ }^{207} \mathrm{Bi}$ conversion electrons which are much more localized in the scintillator material than in the case of the cosmic muons. Fig. 15 shows a timing resolution of

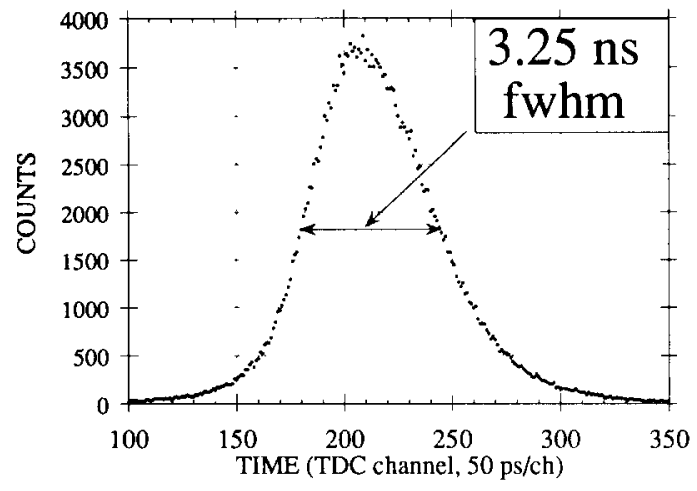

Fig. 13. Timing resolution for $1 \mathrm{MeV}^{207} \mathrm{Bi}$ electrons in a $2 \mathrm{~m}$ long BC414 scintillator with BCF92 fibers.

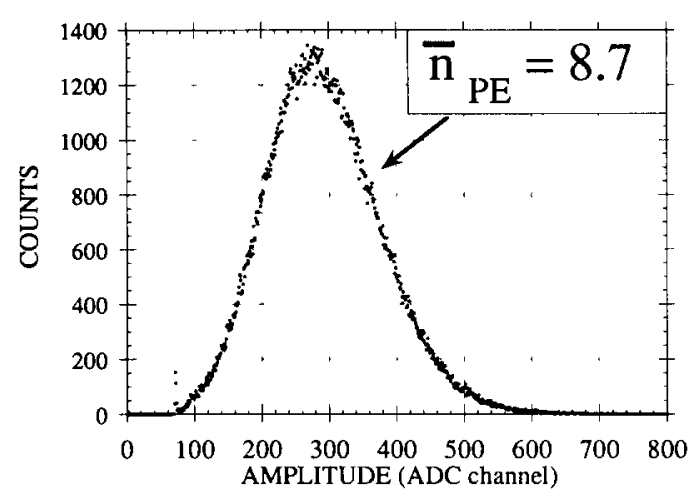

Fig. 14. Number of photoelectrons detected in the measurement from Fig. 13.

$3.75 \mathrm{~ns}$ fwhm obtained in a $4 \mathrm{~m}$ long BC408 scintillator with $6 \mathrm{~m}$ long BCF92 fibers. The cosmic trigger counters defined a $5 \times 10 \mathrm{~cm}^{2}$ spot along the scintillator and an average number of 12 photoelectrons per cosmic particle were detected in the same R580-17 PMT used in all our studies. In a combination consisting of a small piece of $\mathrm{BC} 414$ blue scintillator with $6 \mathrm{~m}$ long

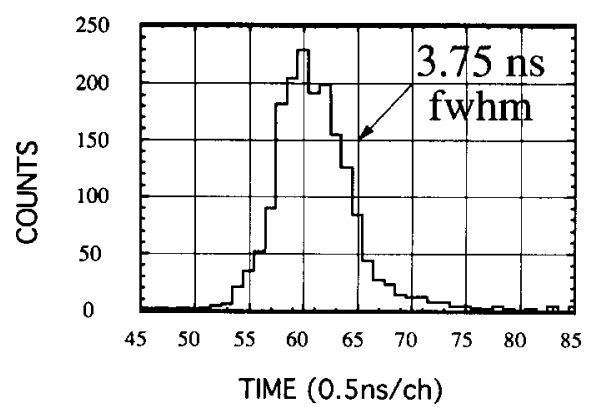

Fig. 15. Timing resolution obtaned with cosmic rays in a $4 \mathrm{~m}$ long BC408 scintillator with $6 \mathrm{~m}$ long BCF92 fibers. An average number of 12 photoelectrons per cosmic particle were detected. 


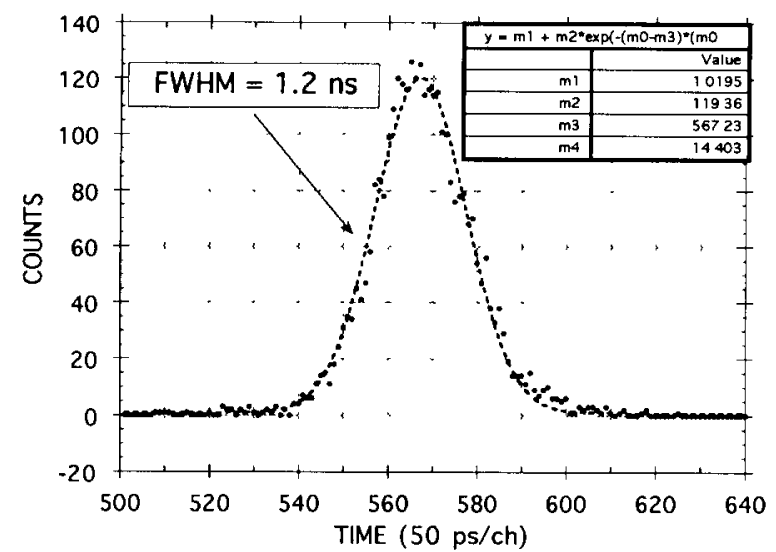

Fig. 16. Timing resolution measured with cosmic rays for a stack of six $1 \mathrm{~m}$ long scint 1 lators wrapped with aluminized

Mylar with five $2.5 \mathrm{~m}$ long BCF92 fibers per scintillator.

BCF99-33A fast blue waveshifting fibers, $4 \mathrm{~ns}$ fwhm was obtained. In a $4 \mathrm{~m}$ long ungrooved $\mathrm{BC} 408$ scintillator with the clear fiber light guide described earlier a result of $3.75 \mathrm{~ns}$ fwhm was obtained showing that indeed BCF92 green waveshifting fibers do not deteriorate timing resolution. In a measurement with a stack of six (four BC414, one BC408 and one BC414) $1 \mathrm{~m}$ long scintillators wrapped individually with aluminized Mylar with five $2.5 \mathrm{~m}$ long BCF92 fibers per scintillator where all thirty BCF92 fibers were coupled to the same R580-17 PMT, a timing resolution of $1.2 \mathrm{~ns}$ fwhm was measured with cosmic rays (Fig. 16) for an average signal of 160 photoelectrons (Fig. 17a). An aluminized Mylar reflector was used on the far scintillator and fiber ends. The average number of photoelectrons detected in this case per one $1 \mathrm{~cm}$ thick scintillator matches exactly the result of 26.5 photoelectrons obtained in the earlier cosmic test with a single $2 \mathrm{~m}$ long BC414 scintillator wrapped with aluminized Mylar with five BCF92 fibers (Fig. 17b). In that measurement the near (PMT side) edge of the scintillator was painted black to allow for only one longitudinal reflection at the far end, adding at most 20 ns to the pulse width (see discussion later). In both cases the secondary peak due to cosmic showers is seen. The high photoelectron signal obtained in these cases is due to a very good match of $\mathrm{BCF} 92$ to $\mathrm{BC} 414$ and includes reflected light from the other end of the fibers with an aluminized Mylar mirror. The matching and mirror issues will be disussed below. Fig. 18 shows two examples of pulses obtained from the stack of six scintillators confirming again that BCF92 fibers are fast.

In some cases another factor can actually give the fiber readout better time resolution. As was pointed out by Kuhlen et al. [12], the limited phase-space in which photons can propagate in the fibers as compared to the bulk scintillator pieces improves the resolution for the same number of detected photons. Also, in our case, near the PMT for the clear fiber light guide there is a higher light output and thus a better resolution. However, after a certain distance, the waveshifting fibers give better resolution because of their longer attenuation length. Fig. 19 shows the dependences of the average number of photoelectrons detected per 1 $\mathrm{MeV}{ }^{207} \mathrm{Bi}$ conversion electron (a) and the corresponding timing resolution (b) in two $4 \mathrm{~m}$ long BC408 scintillator pieces. The light from the grooved scintillator with five $6 \mathrm{~m}$ long BCF92 fibers and wrapped with aluminized Mylar was measured with an R580-17 PMT while a bialkali Philips XP2262 PMT was used with the clear fiber light guide on the ungrooved piece wrapped with a black matte paper and with the far end painted black.

This comparison also demonstrates once again the main advantage of the waveshifting fiber readout and, in general, of waveshifting light collectors. For about the same light output, depending on the particular scintillator/waveshifter combination, wrapping material, reflectors or absorbers used, the active area on the
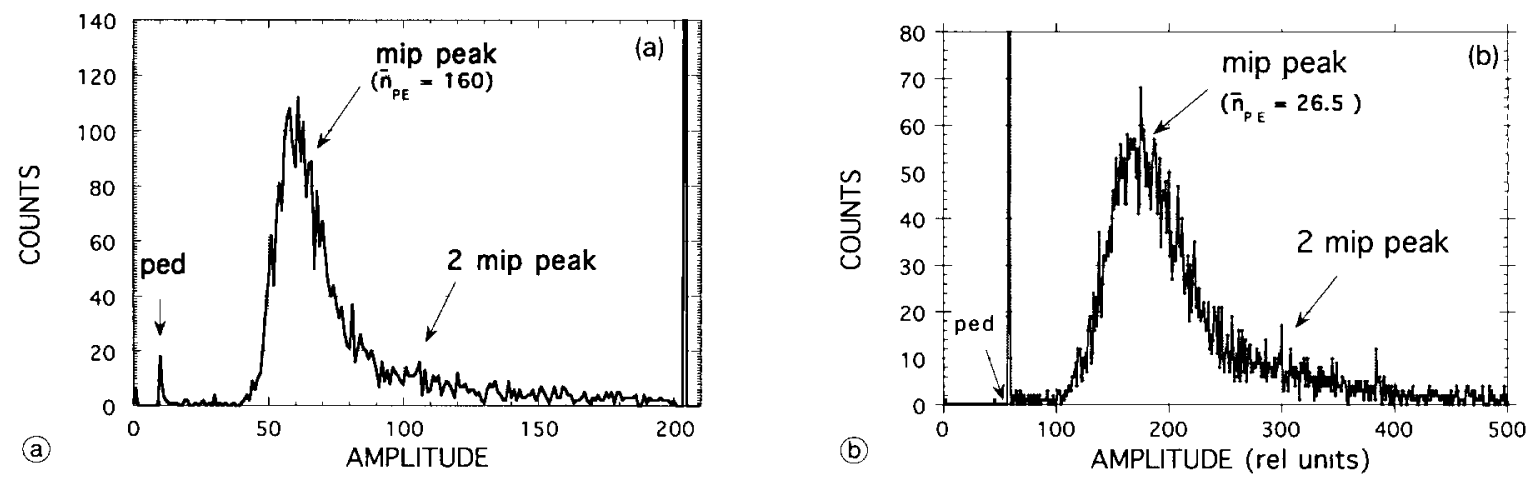

Fig. 17. Photoelectron signal measured for cosmic particles from a stack of six $1 \mathrm{~m}$ long scintillators wrapped with aluminized Mylar with five BCF92 fibers each (a) and for a single $2 \mathrm{~m}$ long BC414 scintillator with five BCF92 fibers (b). 

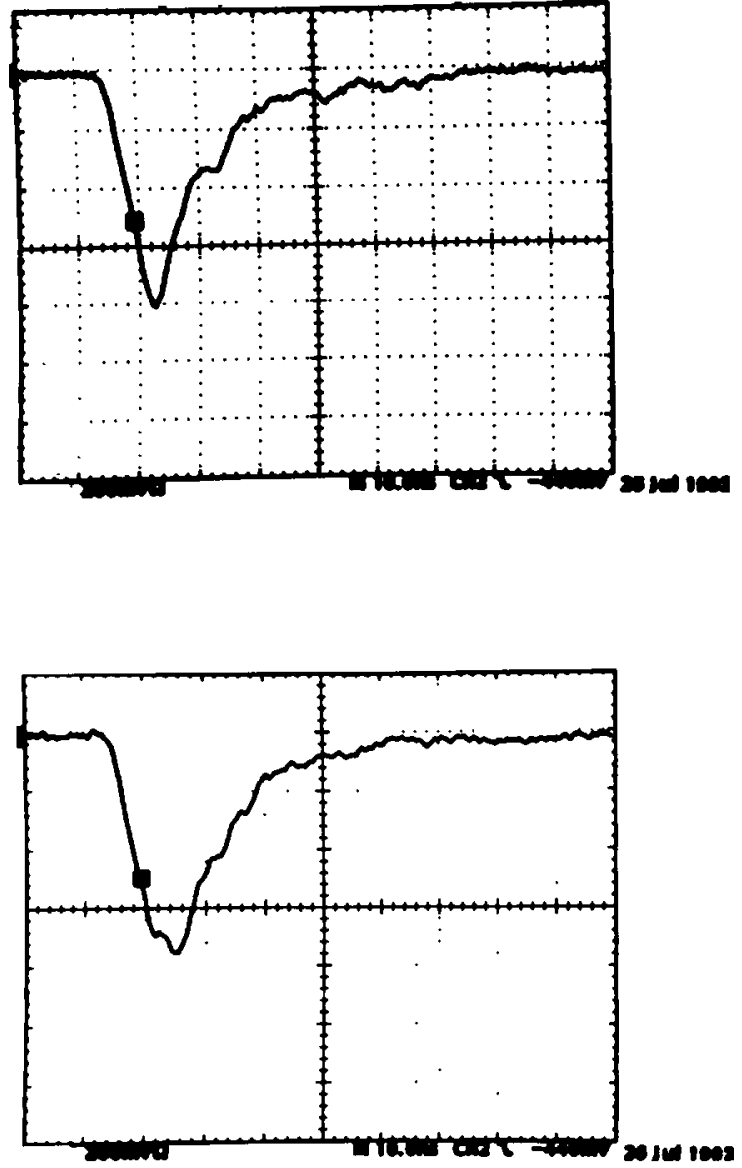

Fig. 18. Two examples of pulses obtained from the stack of six scintillators with BCF92 fibers. Time scale $10 \mathrm{~ns} /$ div.
Table 2

Light-output in photoelectrons per $1 \mathrm{MeV}$ energy deposited for several scintillator-fiber combinations

\begin{tabular}{lllll}
\hline $\begin{array}{l}\text { Fiber } \\
\text { scint. }\end{array}$ & BCF92 & BCF91A & $\begin{array}{l}\text { Kuraray } \\
\text { Y7 }\end{array}$ & $\begin{array}{l}\text { BCF99- } \\
\text { 33A }\end{array}$ \\
\hline BC414 & 6.7 & & & 3.0 \\
BC404 & 6.5 & & & \\
BC408 & 5.3 & 5.2 & 4.9 & \\
SCSN38 & 3.5 & 3.3 & 3.4 & \\
BC412 & 3.7 & & & \\
SCSN81 & 3.0 & 2.5 & 3.2 & \\
\hline
\end{tabular}

photomultiplier surface used is about an order of magnitude smaller in the waveshifting fiber case (twenty 3 $\mathrm{mm}$ diameter clear fibers versus five $2 \mathrm{~mm}$ diameter waveshifting fibers have an almost exact surface ratio of 9).

We tested several scintillator-fiber combinations (Table 2, Fig. 20) of which we found Bicron's BC414BCF92 to give the best results for $2 \mathrm{~mm}$ fibers, about the same as BC404-BCF92 combination. The data in the table and in the figure is referenced to the ${ }^{207} \mathrm{Bi}$ source being $1 \mathrm{~m}$ from the PMT in the center of a 7.5 $\mathrm{cm}$ long sample wrapped with aluminized Mylar using $2.5 \mathrm{~m}$ long fibers with black paint on their far ends. BCF99-33A is a blue waveshifter while the rest shift light into the green region of the visible spectrum. The emission of the G2 green emitting fluor in BCF92 peaks at about $490 \mathrm{~nm}$ and the overlap of its absorption is indeed best with $\mathrm{BC} 404$ and $\mathrm{BC} 414$ emissions [13] (Fig. 21) as confirmed by the experimental results.
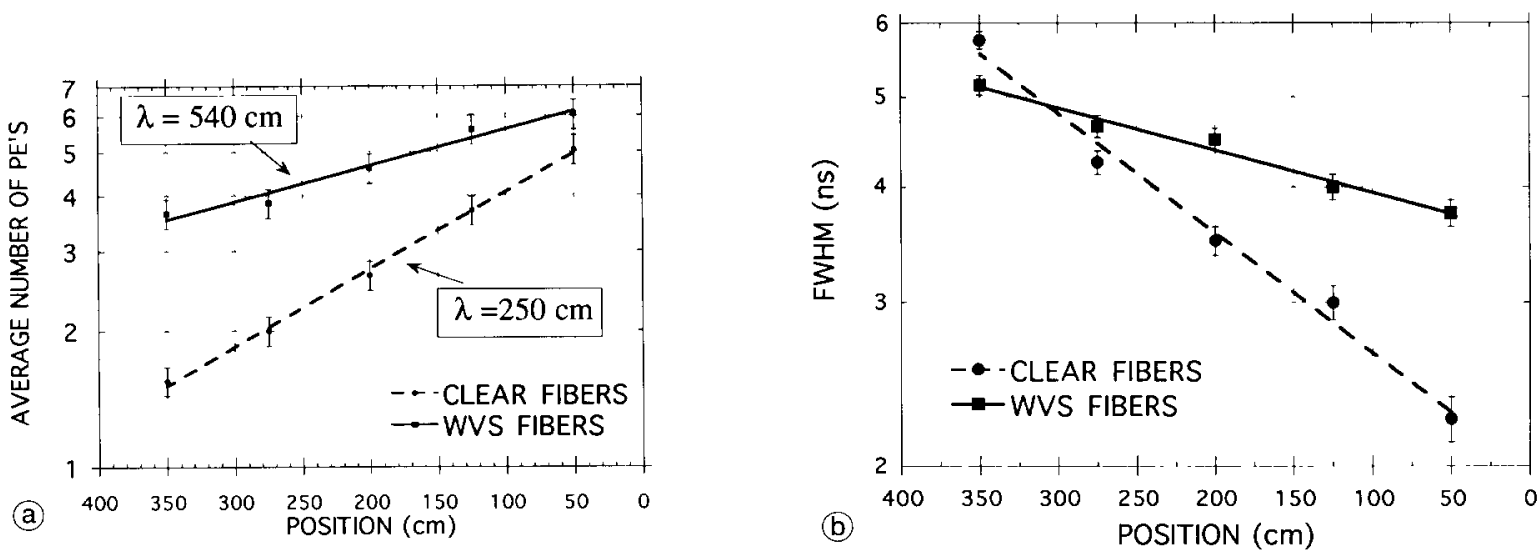

Fig. 19. The dependences of the average number of photoelectrons detected per $1 \mathrm{MeV}{ }^{207} \mathrm{Bi}$ conversion electron (a) and the corresponding timing resolution (b) in two $4 \mathrm{~m}$ long BC408 scintillator pieces. One was grooved, wrapped with aluminized Mylar, used five $6 \mathrm{~m}$ long BCF92 fibers, and was measured with an R580-17 PMT. The second piece was ungrooved, wrapped with a black matte paper with the far end painted black, used the clear fiber light guide, and was measured with a bialkali Philips XP2262 PMT. 


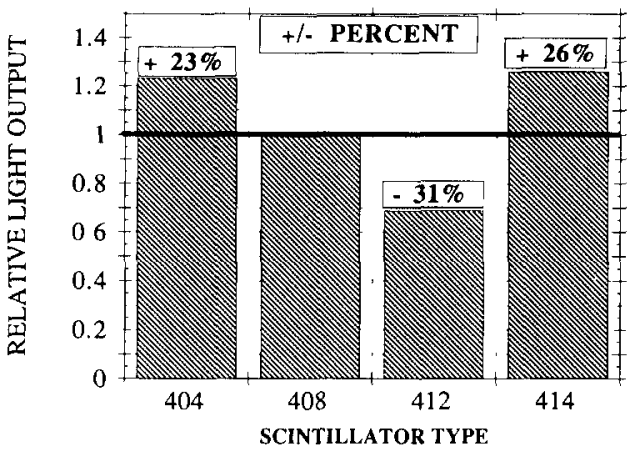

Fig. 20. Relative scintillation signal from four different scintillator pieces of the same size $(1 \mathrm{~cm}$ thick $\times 10 \mathrm{~cm}$ wide $\times 7.5 \mathrm{~cm}$ long) coupled to five BCF92 fibers.

\subsection{Attenuation length}

The attenuation length of the assembly is a combination of the attenuation length of the fibers, which has been measured to be $5-6 \mathrm{~m}$ for its second decay component when excited with UV light (Fig. 22), and the reflective properties of the wrapping and mirror materials. Fig. 23 shows the difference fibers make in the attenuation length of a $4 \mathrm{~m}$ long BC 408 scintillator, increasing it from $260 \mathrm{~cm}$ to $575 \mathrm{~cm}$. Two scintillator pieces were used in these measurements, one grooved with fibers and the second ungrooved. Shown in Fig. 24 (and also in Fig. 22) is the attenuation in a $2 \mathrm{~m}$ long 10 $\mathrm{cm} \times 1 \mathrm{~cm}$ cross section piece of a new green emitting scintillator produced by Bicron with the same G2 waveshifting fluor as used in BCF92 fibers. The measurement was done with an X-ray generator and also shows an excellent attenuation length. The reason the attenuation length is so short near the PMT end of the scintillator appears to be because too high a concentration of the wavelength shifter was used causing self absorption of the emitted light. This was tested by first

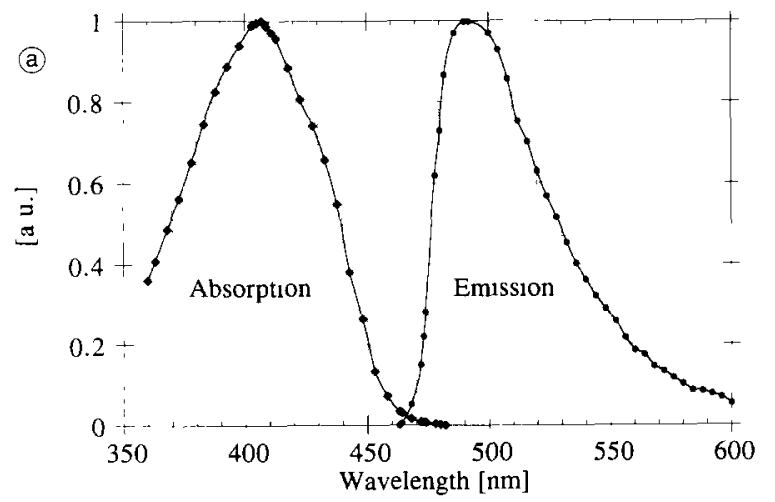

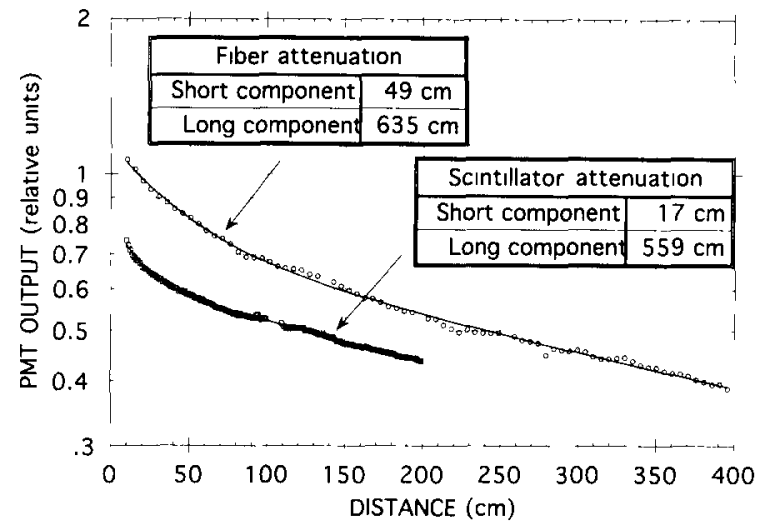

Fig. 22. Attenuation curves measured in BCF92 waveshifting fibers when excited with a UV-light source and in a $2 \mathrm{~m}$ long ( $1 \mathrm{~cm}$ thick $\times 10 \mathrm{~cm}$ wide) piece of a BC-499-29 green emitting scintillator doped with the same waveshıfting fluor when excited with an X-ray beam.

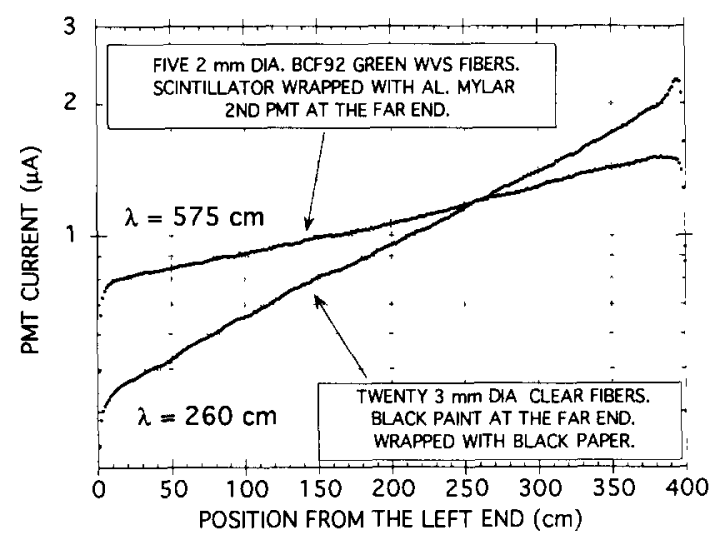

Fig. 23. Attenuation curves for the clear fiber light guide versus waveshifting fiber readout for $\mathrm{BC} 408$.

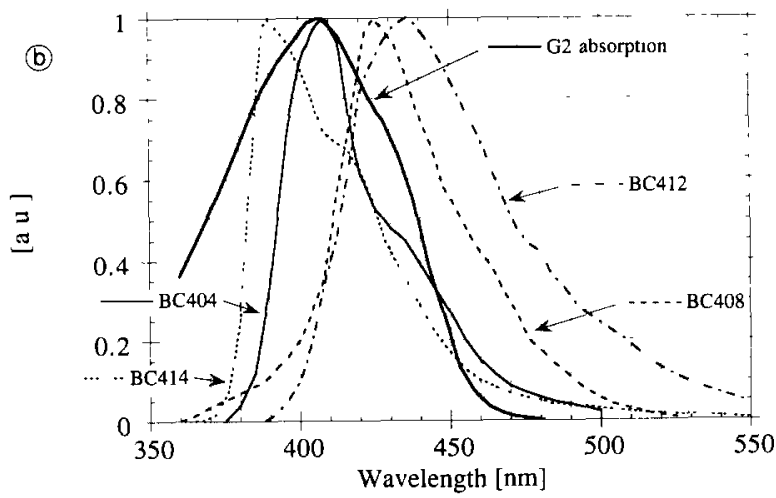

Fig. 21. (a) The absorption and emission spectra for the G2 fluor. (b) A comparison of the G2 absorption and emission spectra for various blue-emitting Bicron scintillators. It shows that BC404 and BC414 have the greatest overlap with the G2 absorption, and hence the best coupling to the BCF-92 WLS fibers for a given intensity. 


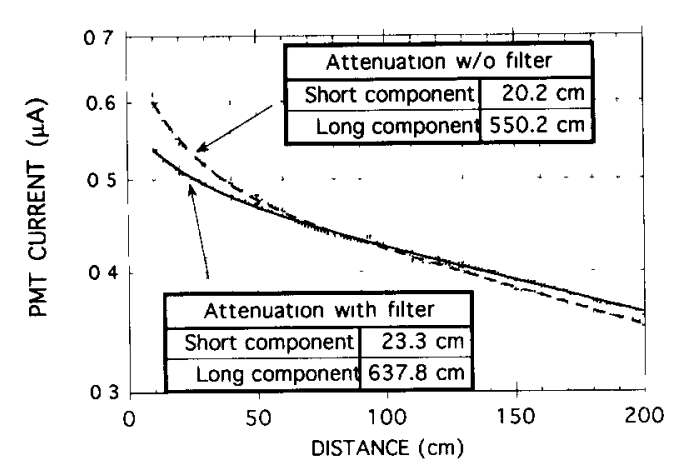

Fig. 24. Attenuation in a $2 \mathrm{~m}$ long $10 \mathrm{~cm} \times 1 \mathrm{~cm}$ cross section piece of a BC-499-29 green emitting scintillator with and without a Kodak Wratten No. 8 green cutoff filter. The curves were normalized in the middle.

using a blue cutoff filter to eliminate the possibility we were measuring unabsorbed blue light and thus had too low a concentration of waveshifter. This produced no change at all. We then used a Kodak Wratten No. 8 green cutoff filter ( $\sim 50 \%$ transmission at $495 \mathrm{~nm}$ ) to cut out the shorter green wavelengths which rid the spectrum of light that would be self absorbed by the waveshifter. This changed the attenuation length to over $6 \mathrm{~m}$ (the second curve in Fig. 24) at a cost of a $\leq 20 \%$ light loss at the $1 \mathrm{~m}$ point.

Another part of our study was testing the effect of mirroring the ends of the fibers and/or scintillator on the attenuation length. Fig. 25a shows the results for various mirroring and absorptive conditions for a $2 \mathrm{~m}$ long BC414-2.5 m BCF92 scintillator-fiber combination. Aluminized Mylar tightly pressed against the fiber and/or scintillator ends or white titanium dioxide reflector paint were used as far end reflectors/diffusers and black paint was used as an absorber. The upper and the lower curves were obtained for an aluminized Mylar wrapping with the scintillator end near the PMT

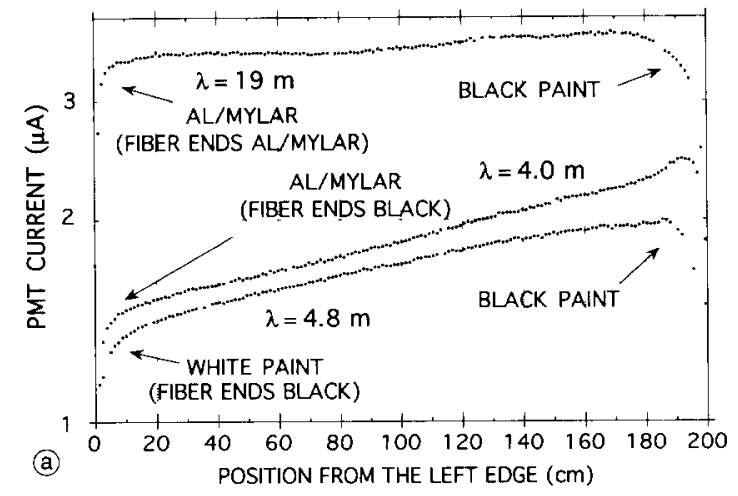

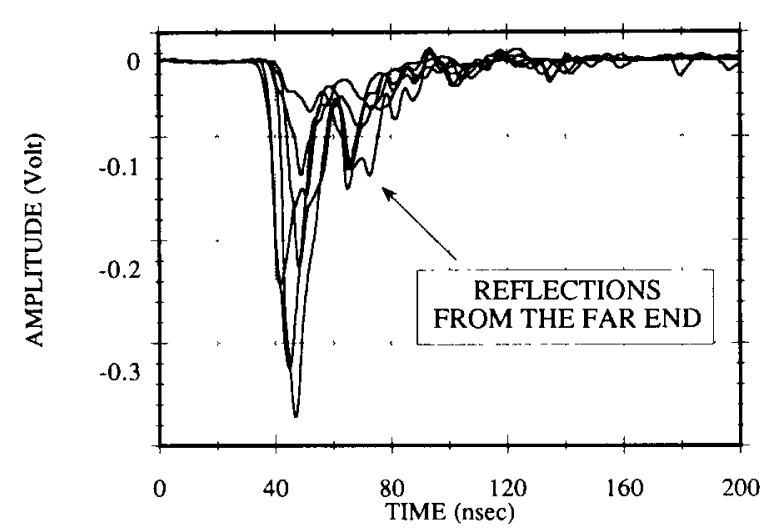

Fig. 26. Picture of cosmic-ray pulses from a 2-m-long BC414 scintillator with 2.5 -m-long BCF92 fibers mirrored with aluminized Mylar at the far end. The cosmic trigger was placed at the PMT end. The reflected pulse at the far end is clearly distinguished.

painted with a black matte paint. The middle curve was obtained when only the fiber ends were painted black. The attenuation measurements were made using a strong ${ }^{106} \mathrm{Ru}$ beta electron source and measuring the PMT current. The attenuation curve fit was made on the data points in the region between $20 \mathrm{~cm}$ to $180 \mathrm{~cm}$. We also made measurements with cosmic rays using the same scintillator piece wrapped with aluminized Mylar and with the aluminized Mylar acting as a mirror at the fiber ends which showed similar results to the current measurement. In Fig. 25b the experimental points from the cosmic measurement were superimposed on the upper curve from Fig. 25a showing good agreement within experimental errors. We took pictures of single pulses using a Tektronix DCS01 digitizing camera system with a Tektronix 2467 oscilloscope. When our cosmic trigger was placed at the near end of a $2 \mathrm{~m}$ long BC414 scintillator with $2.5 \mathrm{~m}$ long mirrored BCF92 fibers, we were able to clearly distinguish the

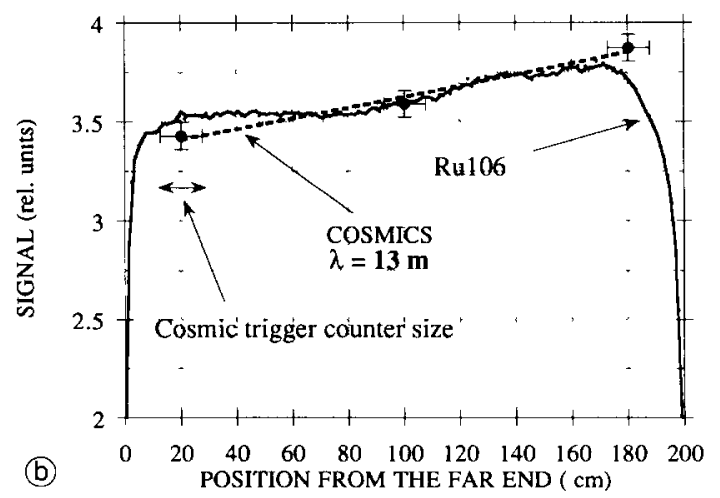

Fig. 25. Attenuation curves for various mirroring and absorptive conditions of the scintillator and of the fibers for a $2 \mathrm{~m}$ long BC414-2.5 m long BCF92 scintillator-fiber combination measured in the current mode (a), and a comparison of the current mode result for the all-aluminized Mylar case (upper curve in (a)) with the result obtained with cosmic rays in the same conditions (b). The PMT was coupled to the fiber ends on the right hand side. 


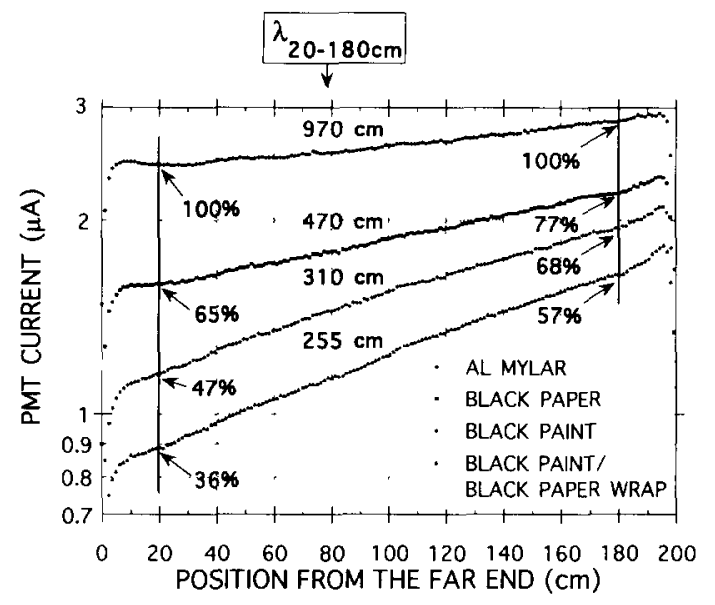

Fig. 27. Attenuation lengths in a $2 \mathrm{~m}$ long ungrooved $\mathrm{BC} 414$ scintillator depending on the wrapping and far end reflectors measured with a collimated ${ }^{106} \mathrm{Ru}$ source and a clear fiber light guide.

reflected pulse which was delayed by about $20 \mathrm{~ns}$ or 5 $\mathrm{ns} / \mathrm{m}$ (Fig. 26). By placing the cosmic-ray trigger at the far end or painting the ends of the fibers black, the reflected pulse disappeared as expected. From these results it is obvious that an efficient reflection at the far fiber ends greatly increases the overall scintillation signal and dramatically improves the apparent attenuation length. If one can afford a long integration time (approximately $70 \mathrm{~ns}+10 \mathrm{~ns} / \mathrm{m}$ length of scintillator) attenuation lengths of $10 \mathrm{~m}$ or more are easily obtained using the reflected light. For even better results, it is possible to sputter aluminum onto the fiber ends, thus creating a high quality mirror [14].

The choice of wrapping and reflecting materials also affects the light output measured in ungrooved scintillator pieces read with the clear fiber light guide. Attenuation lengths between $2.5 \mathrm{~m}$ and almost $10 \mathrm{~m}$ were obtained in a $2 \mathrm{~m}$ long BC414 scintillator depend-

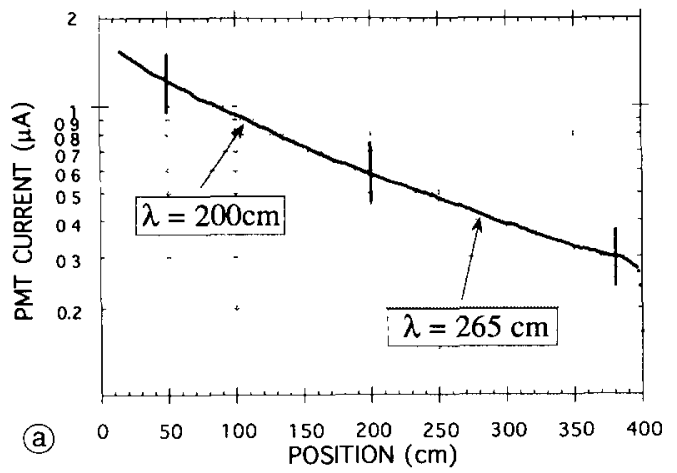

ing on the wrapping and far end reflectors when measured with a collimated ${ }^{106} \mathrm{Ru}$ source (Fig. 27). It is most interesting that changing wrapping from black paper to aluminized Mylar increases light output by about 30\% (the two top curves in Fig. 27). This may be explained by the improved light conversion in the final waveshifter dopant of the scintillator by back reflection of the unconverted short wavelength light trying to escape the active scintillator volume.

In a comparative measurement with a $10 \mathrm{~cm}$ wide 1 $\mathrm{cm}$ thick and $30 \mathrm{~cm}$ long waveshifting bar doped with the same green emitting G2 waveshifting fluor and placed perpendicularly at the end of a $4 \mathrm{~m}$ long ungrooved $\mathrm{BC} 408$ scintillator, one sees the advantage of distributed waveshifting via embedded WLS fibers versus the confined waveshifting due to the WLS bar placed at the end of the scintillator. The scintillator piece was wrapped with black paper and the far end (away from the PMT) was painted with a black paint. The amount of converted light guided to the PMT is higher in the latter case, but a shorter attenuation length, typical of the scintillation material, is obtained as shown in Fig. 28a where the R580-17 PMT was coupled directly to the $1 \mathrm{~cm} \times 10 \mathrm{~cm}$ side of the waveshifting bar. The attenuation length, obtained using a collimated ${ }^{106} \mathrm{Ru}$ source, is even slightly lower than when measured before with the clear fiber readout (Fig. 23). This can be explained by a partial mismatch between the BC408 emission spectrum and the G2 waveshifter absorption (Fig. 21b). The shorter wavelength part of the $\mathrm{BC} 408$ emission which is preferentially converted in G2 undergoes more self-absorption on its way to the scintillator end than the longer wavelengths which are less efficiently converted. As a result the practical absorption length measured with a waveshifting bar gets shortened. Another contributing factor comes from the fact that the waveshifting bar, unlike the clear fiber light guide, does not select the more direct light traversing scintillator which has fewer reflections and, therefore, lower light losses. Clear

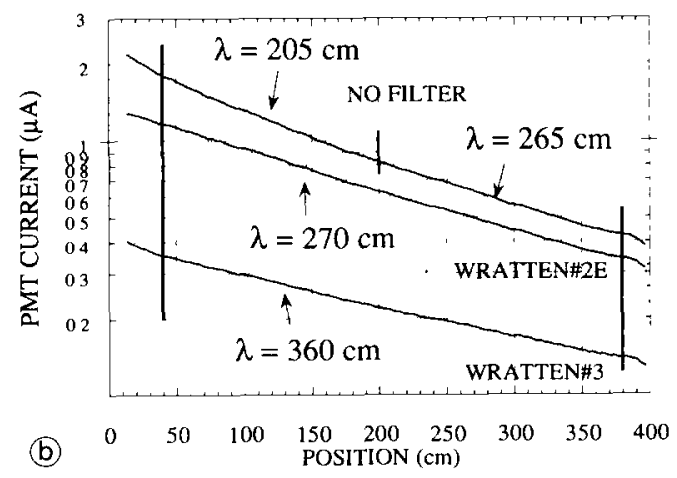

Fig. 28. Attenuation measurements in a $4 \mathrm{~m}$ long ungrooved BC408 scintillator with a G2-doped waveshiftıng bar placed at the scintillator end (a) and a NE172 waveshifting bar with and without Kodak Wratten No. 2E and No 3 filters (b). 
fiber readout has a "narrow vision" angular acceptance due to the limited numerical aperture from the ratio of the refractive indices of its core and cladding materials. A possible remedy is to insert optical filters between the scintillator end and the waveshifting bar, at an expense of lowered light output. Using a different slower ( $\tau=6.5 \mathrm{~ns}) \mathrm{NE} 172$ waveshifting bar from NE Technology [15] with the same $4 \mathrm{~m}$ long BC408 scintillator, an improvement in the attenuation length was obtained going from a two component fit of $205 \mathrm{~cm}$ and $265 \mathrm{~cm}$ in the no filter case to single component fits of $270 \mathrm{~cm}$ and $360 \mathrm{~cm}$ when Kodak Wratten No. 2E and No. 3 filters were used, respectively. The Wratten No. 3 filter cut too much light since its transmission edge is at $465 \mathrm{~nm}$ (@50\% transmission), however a simple single-component attenuation behaviour $(\lambda=$ $270 \mathrm{~cm}$ ) was obtained with a Wratten No. 2E filter with only a small fraction of light lost. No further optimization of the waveshifting bar method was attempted but it is obvious that this old technique might constitute the simplest and most efficient solution when moderate performance (in terms of attenuation length, timing, and light output) is required. Also, in this report we are not attempting a thorough comparison of the waveshifting fiber technique with the clear fiber light guide method. Recent tests with improved BC412 scintillators with the clear fiber light guides routinely yield attenuation lengths better than $3 \mathrm{~m} \mathrm{[16].} \mathrm{However,} \mathrm{the}$ green waveshifting fibers still produce significantly better performance.

\section{Acrylic scintillators}

We have also made many tests with acrylic scintillators which usually suffer from low light output and short attenuation lengths $(\sim 1 \mathrm{~m})$ and are reported to be less radiation hard than polystyrene or PVT based

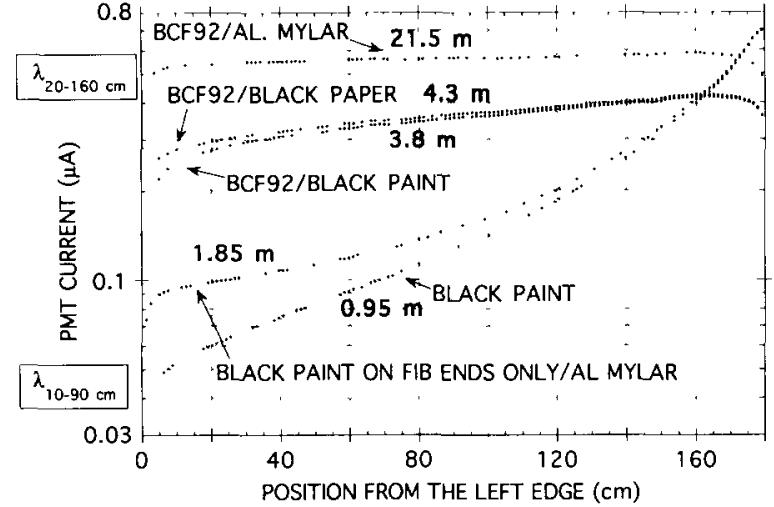

Fig. 30. Attenuation curves obtained for the glued PS10 scintillator module with five imbedded BCF92 fibers. The two lower curves are measured using the clear fiber readout with the light from the waveshifting fibers blocked out (see text for details).

standard plastic scintillators. However they are substantially cheaper than standard scintillators. Figs. 29 and 30 show that the embedded fiber technique easily overcomes attenuation problems in these scintillators. In this case a one quarter inch $(6.4 \mathrm{~mm})$ thick, four in. $(10 \mathrm{~cm})$ wide and $6 \mathrm{ft}(183 \mathrm{~cm})$ long piece of a commercial acrylic-based scintillator Polycast PS10 [17] was grooved with five $2 \mathrm{~mm}$ grooves, the BCF92 fibers were laid in the grooves and the first series of measurements were made. With a clear light guide, the attenuation length measured with the collimated ${ }^{106} \mathrm{Ru}$ source in the ungrooved reference piece was under $1.5 \mathrm{~m}$ independent of the wrapping used (Fig. 29a). In the grooved piece covered with a second ungroved piece (but not glued together) sandwiching the waveshifting fibers, however, the attenuation length reached almost $4 \mathrm{~m}$ (Fig. 29b). In the second series of measurements, the grooved piece was glued to the ungrooved piece with
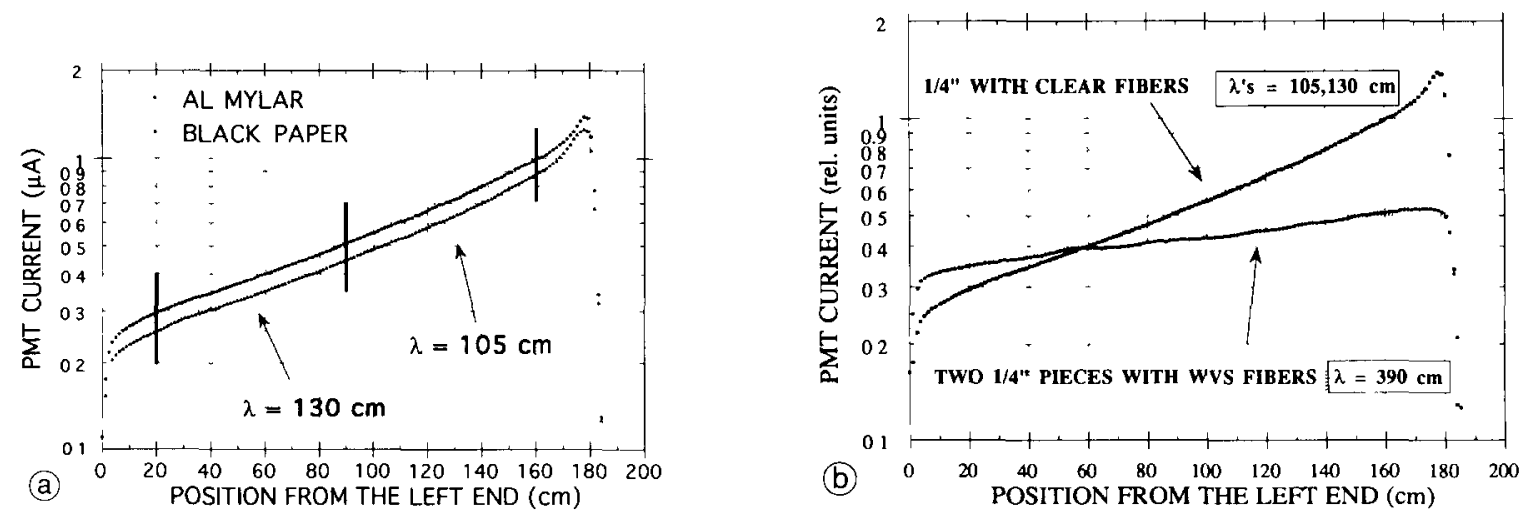

Fig. 29. Attenuation in a one quarter in. $(6.4 \mathrm{~mm})$ thick, four in. $(10 \mathrm{~cm})$ wide and $6 \mathrm{ft}(183 \mathrm{~cm})$ long piece of a Polycast PS10 ungrooved acrylic scintillator (a), and a comparison with the grooved scintillator with five BCF92 fibers covered with a second ungrooved quarter in. thick piece (b). 
Bicron BC600 optical glue with the sandwiched five BCF92 fibers (the fibers were surrounded by glue in their grooves). The attenuation properties of this scintillation module were first studied with a black paper wrapping and then with aluminized Mylar. For comparison, the attenuation length was also measured with the clear fiber light guide, blocking out the light from the imbedded fibers by painting the fiber ends with black paint and coupling the clear fiber light guide directly to the scintillator piece. The two lower curves in Fig. 30 show the results obtained for the aluminized Mylar reflector versus the black paint absorber at the far scintillator end with the fiber ends blocked. The added reflected light from the far end improves the apparent attenuation length in the last section of the scintillator (the left side of the curves in Fig. 30) from about $1 \mathrm{~m}$ to almost $2 \mathrm{~m}$, however, even in this case, the overall light level drops by a factor 7-8 from the near end to the far end of the scintillator module. On the other hand, when measuring the light output from waveshifting fibers and with an alumized Mylar reflector at the far end, the apparent attenuation length can be pushed to over $20 \mathrm{~m}$, as can be seen in the upper curves of Fig. 30.

However, the problem of the relatively low light output of acrylic scintillators still remains. We have tested several types of acrylic scintillators, some of them custom made for us by PolHiTech in Italy [18]. The tested acrylic samples had fast scintillation pulses similar to the ones from a reference NE110 scintillator. The relative light output detected from $1 \mathrm{~cm}$ cube samples with a bialkali RCA $31000 \mathrm{M}$ PMT is $32-43 \%$ of that of NE110 (Fig. 31), however, it is felt that this number can be improved with a better selection of the type and doping level of the scintillating fluor dopants. Also, recently available double-clad waveshifting fibers and better green sensitive PMTs produce an increase in the detected signal from the grooved scintillator

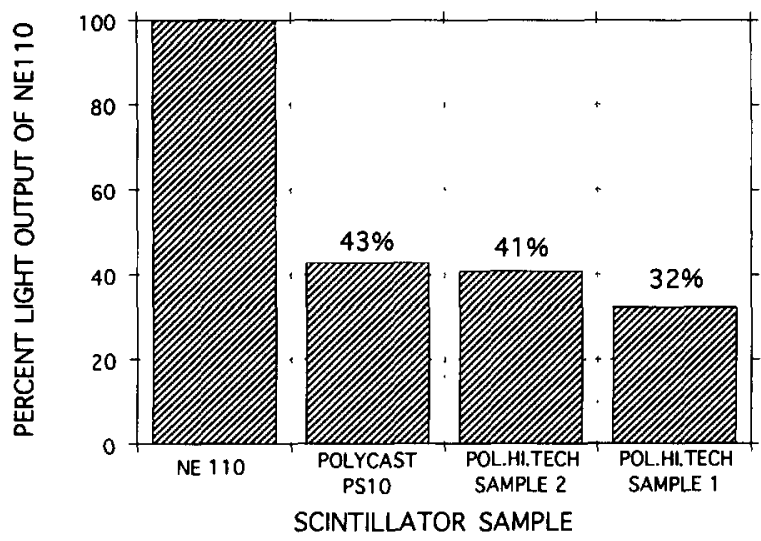

Fig. 31. The relative light output from $1 \mathrm{~cm}$ cube samples of acrylic scintillators measured with a bialkali RCA $31000 \mathrm{M}$ PMT.

modules making the use of acrylic scintillators more practical. For example, using two $3 \mathrm{~m}$ long $1 \mathrm{~mm}$ diameter double-clad Kuraray Y11 fibers [19] per each $2 \mathrm{~mm}$ groove, we obtained 6 photoelectrons per cosmic particle in a $1 \mathrm{~m}$ long $\times 10 \mathrm{~cm}$ wide $\times 1 \mathrm{~cm}$ thick piece of acrylic scintillator wrapped with Tyvek and doped with PTP and bis-MSB as primary and secondary fluors, respectively. The cosmic hodoscope counters were placed in the middle of the scintillator and the converted light had to traverse $1 \mathrm{~m}$ of fiber length on its way to the PMT. The far ends of the fibers were painted black. In the process of attempting to produce improved acrylic scintillators, one material from PolHiTech doped with $12 \%$ of naphtaline, $2 \%$ butyl-PBD and $0.05 \%$ of BDB came with a very short attenuation length. This heavily doped formula, called sample 2 in Fig. 31, was based on the original formula used in a Kuraray SCSN38 scintillator [19] and resulted in a slight pink discoloration of the material. The curve in
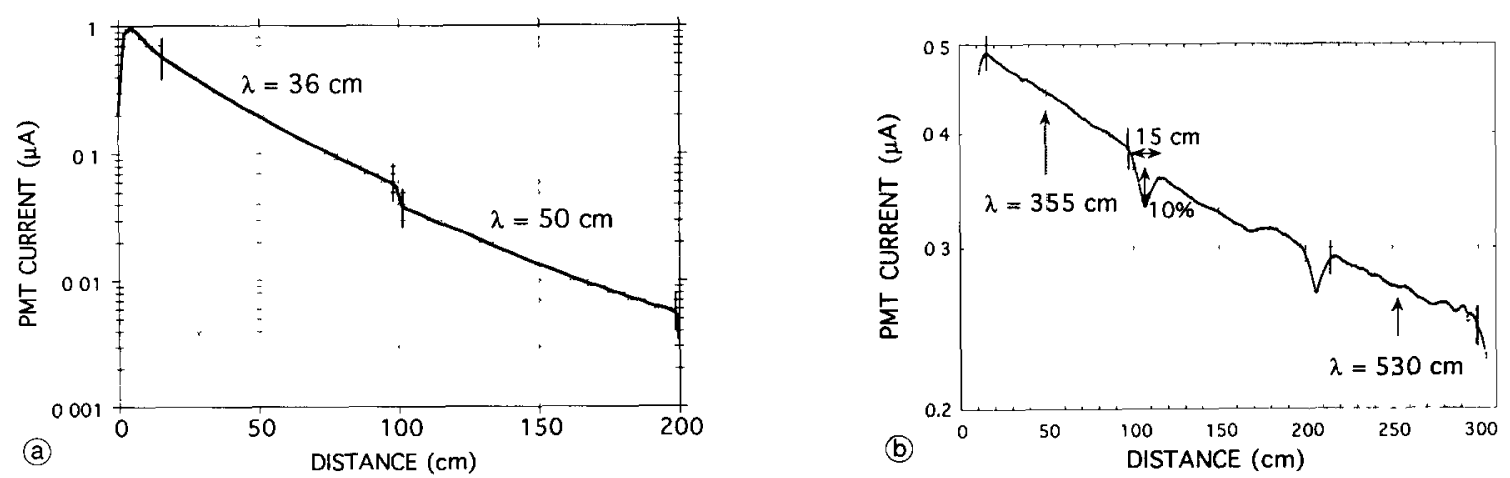

Fig. 32. The result of a longitudinal scan with an X-ray beam for two abutted ungrooved $1 \mathrm{~m}$ long pieces of a PolHiTech acrylic scintillator with a clear fiber light guide and a black paper wrapping (a), and for five $6 \mathrm{~m}$ long $2 \mathrm{~mm}$ diameter BCF92 waveshifting fibers placed in three grooved and abutted $1 \mathrm{~m}$ long pieces of the same scintillator. 
Fig. 32a shows the result of a longitudinal scan with an $\mathrm{X}$-ray beam for two ungrooved $1 \mathrm{~m}$ long pieces of this scintillator butted together. The clear fiber light guide was used and the scintillator was wrapped with black paper. Indeed, a very short attenuation length was measured with a light loss at the junction. A dramatic improvement in attenuation was obtained with five $6 \mathrm{~m}$ long $2 \mathrm{~mm}$ diameter BCF92 waveshifting fibers placed in three grooved $1 \mathrm{~m}$ long pieces butted together (Fig. $32 b)$.

This example makes the best experimental demonstration yet of the intrinsic insensitivity of the waveshifting fiber method to the transmission properties of the bulk material. It also implies that radiation-induced transmission damage of the grooved scintillator modules will be almost entirely defined by the damage to the fibers. In consequence, it might be possible to employ acrylic scintillators, which are known to be less radiation hard than polystyrene or polyvinyltoluene based standard plastic scintillators, in high radiation dose applications. The remaining problem is the $\sim 10 \%$ amplitude decrease in a $7-8 \mathrm{~cm}$ wide region close to the ends of the scintillator pieces, which is especially visible in the butting regions (Fig. 32b). The importance of this effect depends on the particular application and, in principle, can be corrected by a technique developed for the ZEUS electromagnetic calorimeter where the reflectivity of the wrapping material was gradually changed to compensate for the edge effects [9].

\section{Photomultipliers}

The majority of our tests were made using the Hamamatsu R580-17 green extended PMT which was found to have $20 \%$ to $50 \%$ more quantum efficiency than standard bialkali PMTs for the green light from the fibers, as well as having a good single photoelectron (SPE) response. The Burle S83010E [20] and EMI $9902 \mathrm{~KB}$ [21] were also good, however, they were found to be more susceptible to the Earth's magnetic field. We also obtained good results with the Russian FEU115M [22]. Table 3 shows a comparison of the different PMTs we tried with green waveshifting fibers. As seen, the Hamamatsu R4760 multianode (16 channel) PMT has about the same quantum efficiency as standard bialkali PMTs and with a green extended photocathode might be an interesting compact solution in the readout of many waveshifting channels, provided the diameter of each fiber bunch is less than $\sim 5 \mathrm{~mm}$ and the slow (6-7 ns) rise time of this PMT is acceptable. A cross talk between neighbouring channels of less than $0.8 \%$ was measured in this PMT [23]. We also tried inexpensive side-window PMTs such as the 931B but found that the geometrical factors of the photo cath-
Table 3

Comparison of sensitivity of different PMTs in the detection of light from green waveshifting fibers

\begin{tabular}{ll}
\hline Tube type & Relative sensitivity (\%) \\
\hline Hamamatsu R580-17 & 100 \\
Burle S83010E & 94 \\
Russian FEU-115M & 93 \\
Thorn-EMI 9902KB & 87 \\
Hamamatsu R2083 & 83 \\
Philips XP2081B & 83 \\
Hamamatsu R4760 & $57^{\text {a }}$ \\
Hamamatsu R2368 & $57^{\text {b }}$ \\
Hamamatsu R2165 & $52^{2}$ \\
RCA 31000M & $47^{6}$ \\
Hamamatsu R1332Q & $46^{\text {b }}$ \\
Hamamatsu R3788 & $26^{\text {b }}$ \\
Hamamatsu R2693 & $22^{\text {b }}$ \\
Hamamatsu 931B & $22^{\text {b }}$ \\
\hline
\end{tabular}

a Multianode PMT.

b Includes geometrical factors (side-window, small size PMT).

ode limited their practical efficiency to about half that of standard bialkali PMTs [24].

\section{Applications}

In applying our technique to detectors to be used at CEBAF, we built three cosmic ray hodoscopes for the tests of lead glass blocks with counter sizes from $8 \mathrm{~cm}$ to $100 \mathrm{~cm}$ and with three to five fibers per scintillator. We used a Hamamatsu R4760 16 channel PMT and sixteen inexpensive small side-on Hamamatsu 931B PMTs to read the waveshifting fibers. By having the side-on tubes preselected by the manufacturer to have the same gain within $20 \%$, we were able to use a single power supply to power them. In spite of their lower effective QE the signal from the side-on PMTs produced by cosmic muons passing through the $1 \mathrm{~cm}$ thick hodoscope scintillators was high enough to enable efficient, close to $100 \%$, cosmic ray detection.

\section{Grooving}

Grooving technique is of primary importance if the proposed readout method is to be practical. The published material [25] on grooving scintillators suggests using single-edge cutting tools run at high speed for the optimum finish. Our first efforts to duplicate this produced unsatisfactory results due to localized melting of the material in the gronve. The low thermal conductivity of the plastic made it nearly impossible to cool effectively. This was further aggravated by the scintillator's sensitivity to damage from solvents or oils, 
precluding the use of most conventional cutting fluids. Polystyrene and acrylic based materials are notoriously sticky. The chips tend to adhere to the cutter, which then drags them through the groove causing a poor finish. After testing several cutting fluids, the optimum was found to be a $50 / 50 \mathrm{mix}$ of clean water and mild dish washing soap sprayed through an air mister. This mix prevented the chips from sticking to the cutter and the air mister forced them out of the way.

Several cutter geometries were evaluated. We attempted using a commercial high-speed steel convex milling cutter (similar to a slitting saw) which gave better results than the single-edge tools. To improve on that, we ordered [26] a similar cutter made entirely of micro-grain carbide (Fig. 33a). We found the higher the speed the tool was run at, the finer the finish. We ran it at 3000 revolutions per minute, the limit for this type of tool. The feed rate reached its optimum at 4 inches per minute (IPMT). Even a small increase to 6 IPMT caused degradation of the finish. Using this type of cutter allows multiple cutters to be ganged so all grooves can be cut in a single pass (Fig. 33b). These cutters leave a transparent finish almost as smooth as cast finishes on commercial scintillators, but with some light diffusing quality which is claimed to improve waveshifting operation $[10,25]$. We found that this grooving method is easily transferred to industry and have, in fact, had most of our scintillators grooved by an outside machine shop.

Another method of producing grooved scintillators is to extrude them. A grooving nozzle (Fig. 34) produced at CEBAF has been successfully used to produce good mechanical quality extruded grooved $1 \mathrm{~cm}$ thick by $10 \mathrm{~cm}$ wide and up to $3 \mathrm{~m}$ long polystyrene scintillators in Yerevan, Armenia [27]. In this method the surface of the grooves is as smooth as in other flat regions of the sample. Due to poor matching to the

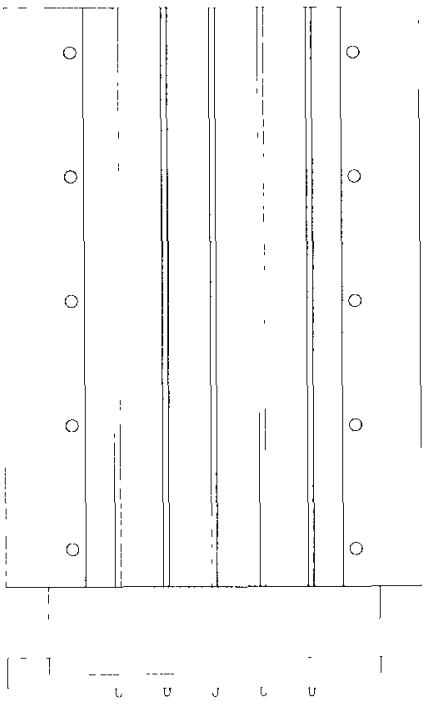

Fig. 34. A drawing of a grooving nozzle used to produce extruded grooved $1 \mathrm{~cm}$ thick $10 \mathrm{~cm}$ wide and up to $3 \mathrm{~m}$ long polystyrene scintillators.

waveshifter used in the scintillator material itself, the scintillator-fiber combinations suffered from relatively low light output, giving only about 2 photoelectrons per $\mathrm{MeV}$ with the source $50 \mathrm{~cm}$ from the PMT end of the scintillator and a fiber length of $4 \mathrm{~m}$. Also, light selfabsorption due to impurities present in the material produced a short attenuation length when measured with the clear fiber light guide. However, as in the case of acrylic scintillators, long attenuation lengths typical of waveshifting fibers were obtained when the waveshifting fiber readout was used (Figs. 35 and 36). The transmission curve from Fig. 35 obtained with the clear fiber light guide in a $187.5 \mathrm{~cm}$ long grooved module wrapped in black paper without embedded
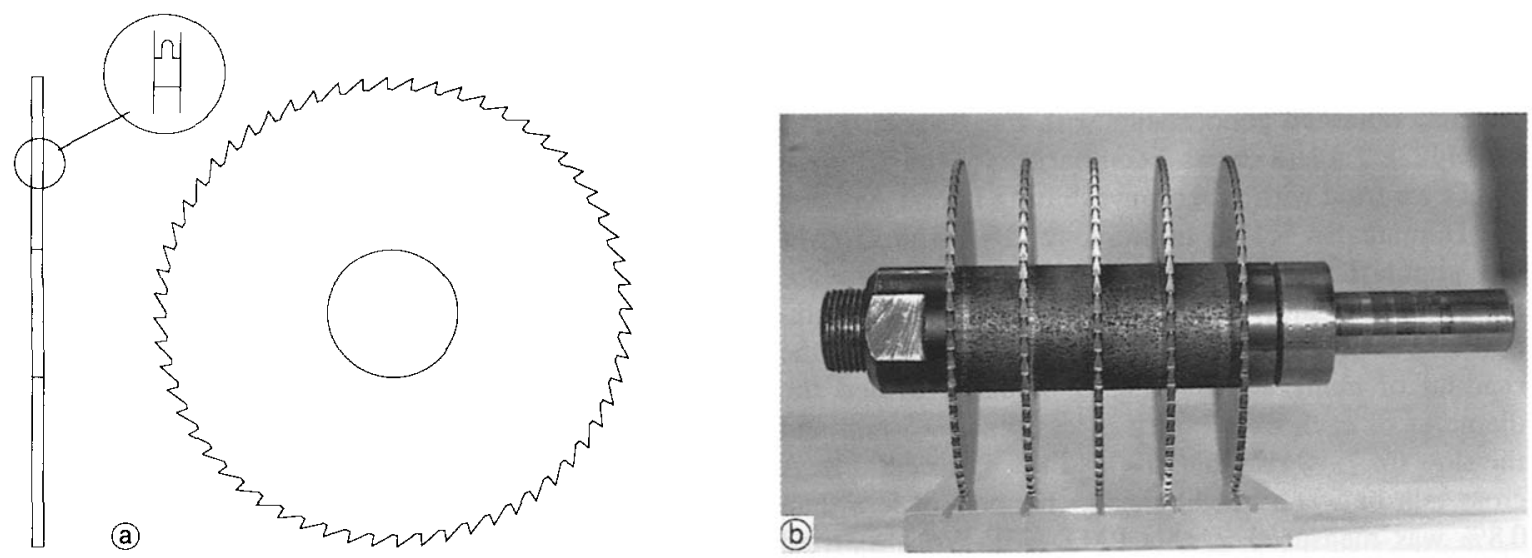

Fig. 33. A schematic of a single micro-grain carbide cutter (a) and a picture of the tool used with five cutters ganged together so that all five grooves can be cut in a single pass (b). 


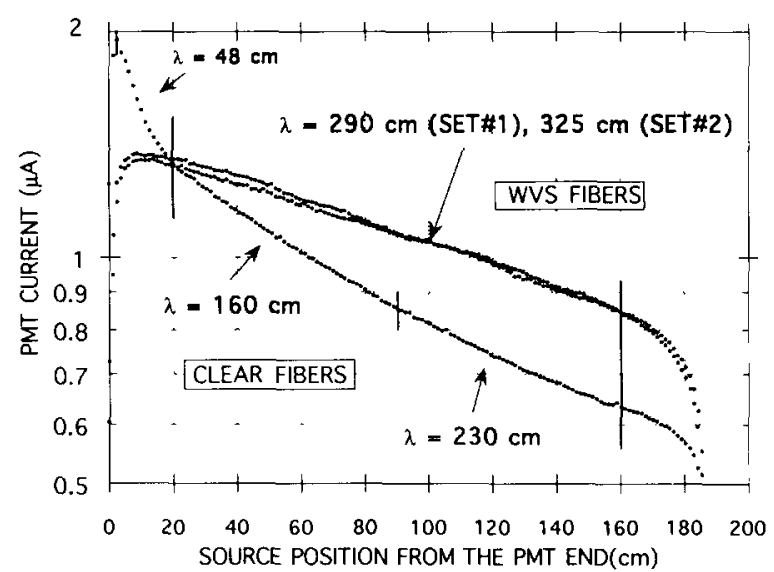

Fig. 35. Transmission curves obtained with the clear fiber light guide of a $187.5 \mathrm{~cm}$ long grooved extruded module wrapped in black paper without waveshifting fibers and for the same grooved module wrapped with aluminized Mylar for two $2.5 \mathrm{~m}$ long BCF92 fiber sets.

waveshifting fibers shows three attenuation components with $\lambda$ values from $48 \mathrm{~cm}$ to $260 \mathrm{~cm}$ and, therefore, a very poor uniformity along the scintillator. The difference seen for two $2.5 \mathrm{~m}$ long fiber sets with the same grooved module wrapped with aluminized Mylar shown in Fig. 35 was traced to scratches on the often used set of waveshifting fibers (set No. 1) and underscores the importance of proper fiber handling. Also, the result depends on the distance the converted light has to traverse in the fibers before entering the PMT. When $6 \mathrm{~m}$ long fibers were used with the $2 \mathrm{~m}$ end sections imbedded in the scintillator module a lower light output was obtained but the average attenuation length improved from above $3 \mathrm{~m}$ to almost $5 \mathrm{~m}$ when

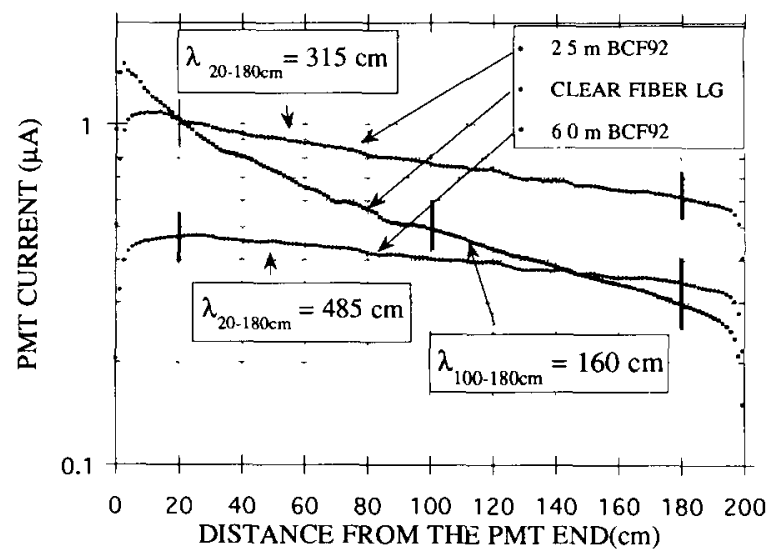

Fig. 36. Comparison of transmission curves in grooved extruded modules measured with the clear fiber light guide, and with five $2.5 \mathrm{~m}$ and five $6 \mathrm{~m} \mathrm{BCF92}$ waveshifting fibers with their $2 \mathrm{~m}$ long end sections imbedded in the scintillator module and wrapped with aluminized Mylar.

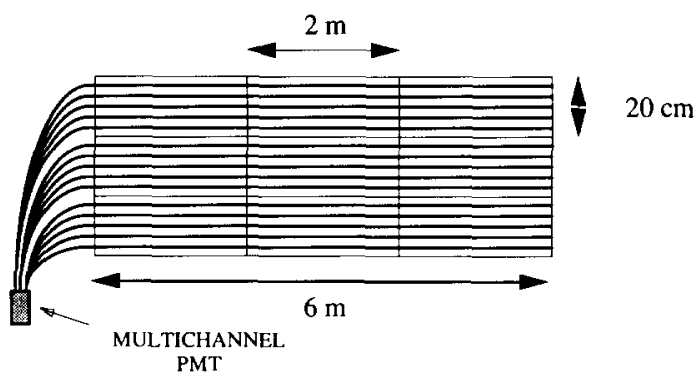

Fig. 37. An example of a large-area scintillator constructed by butting end to end shorter grooved scintillators and reading the light with waveshifting fibers.

compared to 2.5 long fibers (Fig. 36). This result is consistent with the attenuation curves measured for fibers alone (Fig. 24). It would be highly desirable if the grooving-by-extrusion technique were also offered by scintillator manufacturers since this would greatly enhance attractiveness of the waveshifting readout by avoiding the need for the time consuming and delicate grooving operation.

\section{Modular detectors}

Grooved scintillators with fibers can easily be used to construct large-area scintillators by butting shorter scintillators end to end (Fig, 37). This can result in cost savings since shorter scintillators (up to $2 \mathrm{~m}$ ) tend to be about half the cost per meter of longer scintillators $(\sim 4 \mathrm{~m})$. Butting scintillators can also be used to overcome the problem that no company has the facilities to produce scintillators of $5 \mathrm{~m}$ or longer. A few different methods were used to butt the scintillators - air, a 1 $\mathrm{mm}$ thick BC634 soft silicone insert [7], a Tyvek insert, and an aluminum foil insert between the scintillators. Our best results came from air butting, however, more

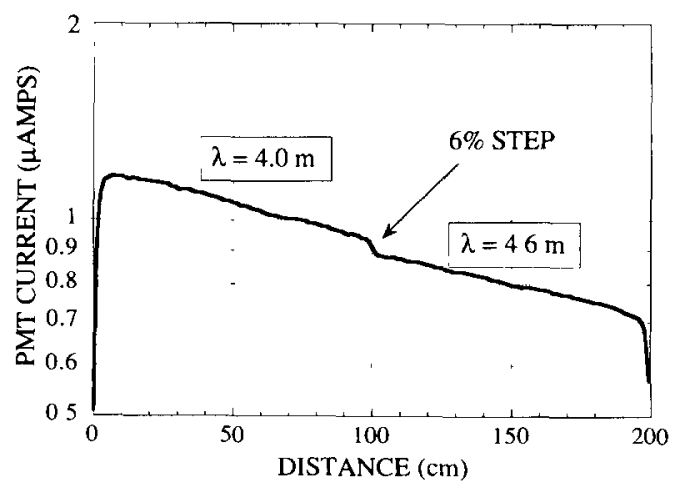

Fig. 38. The loss of light across the air butt joint for two $1 \mathrm{~m}$ long $\mathrm{BC} 408$ scintillators wrapped with aluminized Mylar and read with BCF92 fibers. 
in-depth studies are needed to optimize this technique. The loss of light across the butt joint when using standard scintillators is relatively small (Fig. 38) and can be accommodated in a calibration curve. The edge effect discussed in the section on acrylic scintillators (and shown in Fig. 32b) which is present to a lesser extent in polystyrene or PVT based scintillators needs more study, including computer simulations, to evaluate if it can be corrected for in the calibration curve or if it needs an actual hardware correction similar to the aforementioned method used in the ZEUS calorimeter [9]. One could build, in principle, just about any shape and size of detector out of a few modular scintillator parts (or a "kit"). It would be also desirable if grooved modules could be acquired directly from the scintillator manufacturer.

\section{New developments}

As mentioned before, there is work going on to use multi-clad (double-clad) waveshifting fibers to increase light output. Currently, there are only $1 \mathrm{~mm}$ double clad fibers available doped with a slow green fluor (Y11) from Kuraray [19]. Using two of these $1 \mathrm{~mm}$ fibers per groove, we obtained about a $33 \%$ improvement in the light output over $2 \mathrm{~mm}$ BCF92 fibers independently of the scintillator used (Fig. 39 shows an example for a $2 \mathrm{~m}$ long BC404 scintillator). With one 1 $\mathrm{mm}$ Y11 fiber per groove we found only 3\% less light than with the $2 \mathrm{~mm} \mathrm{BCF92} \mathrm{fibers.} \mathrm{The} \mathrm{attenuation}$ length of the scintillator-fiber sample was found to improve by about $2 \%$ using the double clad fibers. In 1 mm 3HF-doped fibers an improvement of $70 \%$ in the light output was reported in the double clad fibers as compared to the standard fibers doped with the same concentration of $3 \mathrm{HF}$ [28]. We expect a similar improvement for $2 \mathrm{~mm}$ fibers if double clad $2 \mathrm{~mm}$ fibers

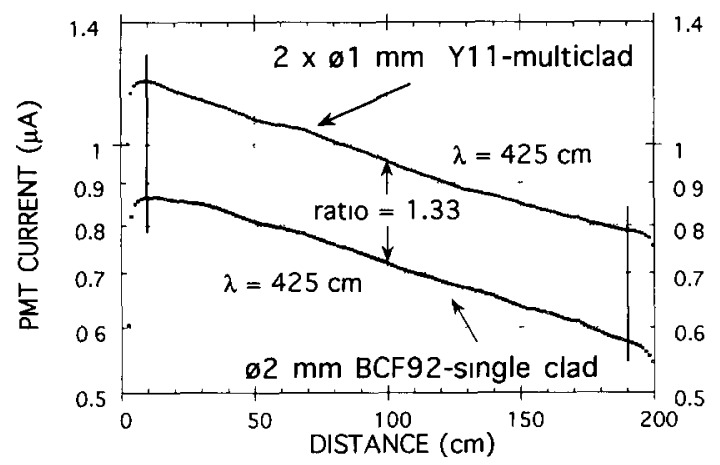

Fig. 39. Comparison of response from a $2 \mathrm{~m}$ long grooved BC404 scintillator using two Kuraray $1 \mathrm{~mm}$ Y11 double clad fibers per groove versus one Bicron $2 \mathrm{~mm}$ BCF92 fiber per groove.

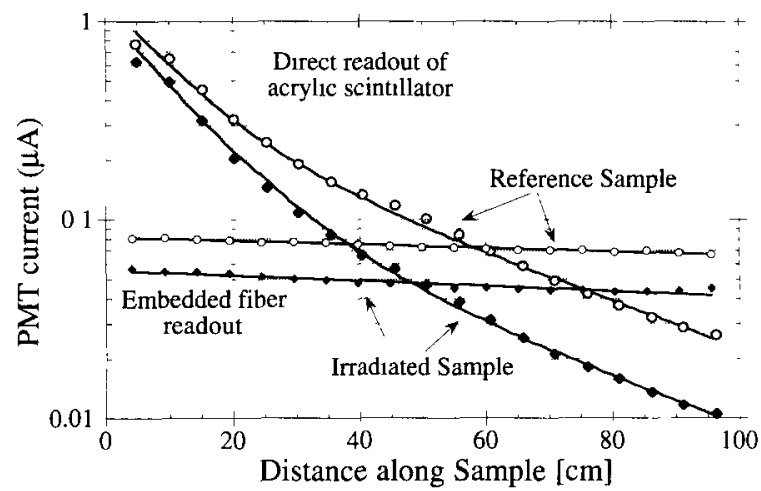

Fig. 40. Comparison of direct vs embedded fiber scintillator readout of radiation damaged acrylic scintillator.

doped with G2 fluor were available from Bicron. The preliminary results show that the double clad fibers are as radiation hard as standard single clad fibers [29].

There is also a proposal to increase the percent of light transmitted down the fiber by non-uniform doping with a waveshifter [30]. It was pointed out in several studies that a higher fraction of the light is trapped when it is produced off-axis rather than on-axis in the fiber. The usually quoted number is calculated for the light emitted from a point located on the fiber axis. The increase of over a factor two for the outer emission points, close to the cladding, is due to spiralling rays captured in the fiber by total internal reflection. For example, in the single clad acrylic-onpolystyrene fiber the capture fraction of $3.14 \%$ (each way) increases to about $7 \%$ for emission points close to the cladding. In the double clad Kuraray fibers the capture fraction for the central location increases to $5.34 \%$ [28] and an increase to about $12 \%$ is expected for the outer rays. Of course, it has yet to be seen how the spiralling trajectories would change attenuation properties of fibers.

\section{Radiation damage}

A low rate, low dose irradiation study was performed on the acrylic scintillator sample 1 from PolHiTech and the BCF92 fibers [31]. After 16 krads in oxygen at $55 \mathrm{rads} / \mathrm{hr}$, the attenuation length of the scintillator is heavily affected whereas, with fibers, there is very little change (Fig. 40). The only affect is an overall loss of light from the scintillator-fiber combination which is much easier to calibrate for than a change in attenuation length. At this dose there is no radiation damage to the fibers. At doses where standard PVT or polystyrene scintillators would show damage, BCF92 would also show radiation damage. In this case, one might just replace the fibers, which, if they are not 
glued in the grooves is a very easy process and can be completed without even unwrapping the scintillators. If one does not need the fast decay time of BCF-92, then Y11 and BCF91 wavelength shifting fibers are suitable radiation tolerant substitutes.

\section{Summary}

We have used successfully the well-known waveshifting fiber readout method to read light from long scintillator pieces. The embedded fiber technique can be used to obtain attenuation lengths of greater than $10 \mathrm{~m}$ even in inexpensive acrylic and otherwise poor quality scintillators with a small number of readout elements-fibers, resulting in a relatively small required active PMT surface, about an order of magnitude smaller than for the same amount of light detected when a clear fiber light guide is used. Light output is lower than for a direct coupled PMT; however, it is sufficient for many applications such as shower counters or hodoscopes when timing resolution is not crucial. Timing resolution obtained with the fast green $\mathrm{G} 2$ fluor in Bicron BCF92 waveshifting fibers matches the resolution obtained with clear lightguides for the same number of photoelectrons detected. Gluing of fibers increases light output but is not necessary; not gluing the fibers makes fabrication a rather simple task and avoids problems with possible aging effects of the glue. Many detector geometries can be produced from a few standard grooved scintillator sizes making mass production attractive. A grooving technique applicable to mass production was developed and tested successfully in realistic conditions. As shown, if the fibers are radiation hard, they make an embedded fiber detector much more radiation hard than a detector depending only on the scintillator due to an increased absorption of the shorter wavelengths in a radiation damaged scintillator. An embedded waveshifting fiber detector loses light output mostly due to the local damage to the scintillation yield, however, it retains its long attenuation length. All major technical difficulties such as grooving have been solved and working detectors have been produced, making this a well-established technique.

\section{Acknowledgements}

We thank Dr. Kim Egiyan and Dr. Stepan Stepanyan from the Yerevan Institute of Physics in Armenia for providing us with the extruded scintillator samples. We express gratidude to Dr. Vasken Hagopian and Maurizio Bertoldi from Florida State University for providing assistance with the evaluation of our grooving technique. Visiting high school students, Blair
Seaford and Paul Sun from the New Horizons Governor's School in Hampton, VA, are thanked for helping with some measurements.

\section{References}

[1] H. Blumenfeld et al., Nucl. Instr. and Meth. A 225 (1984) 518 ; H. Burmeister et al., Nucl. Instr. and Meth. A 225 (1984) 530 ;

H. Blumenfeld et al., Nucl. Instr. and Meth. A 235 (1985) 326;

P. Sonderegger, Nucl. Instr. and Meth. A 257 (1987) 523;

R. DeSalvo et al., Nucl. Instr. and Meth. A 279 (1989) 467;

A. Bross et al., Nucl. Instr. and Meth. A 286 (1990) 69; D.R. Wood, Nucl. Instr. and Meth. A 289 (1990) 331;

A.J. Rusi el Hassani et al., Nucl. Instr. and Meth. A 290 (1990) 109;

S.J. Alvsvåg et al., Nucl. Instr. and Meth. A 290 (1990) 320 ;

D. Hertzog et al., Nucl. Instr. and Meth. A 294 (1990) 446;

D. Acosta et al., Nucl. Instr. and Meth. A 294 (1990) 193; F. Takasaki et al., Performance of Scintillation Fiber Calorimeter and Readout System with Multi-Anode Photomultiplier Tubes, KEK preprint 89-183, January 1990; W. Worstell et al., Proc. Symp. on Detector Research and Development for the SSC, October 15-18, 1990, Fort Worth, Texas, p. 428;

D. Acosta et al., Nucl. Instr. and Meth. A 302 (1991) 36; D. Acosta et al., Nucl. Instr. and Meth. A 305 (1991) 55;

C. Bini et al., Nucl. Instr. and Meth. A 306 (1991) 467;

D. Acosta et al., Nucl. Instr. and Meth. A 308 (1991) 481;

D. Acosta et al., Nucl. Instr. and Meth. A 309 (1991) 143;

T. Arima et al., Nucl. Instr. and Meth. A 314 (1992) 417;

D. Acosta et al., Nucl. Instr. and Meth. A 314 (1992) 431;

R. Wigmans, Nucl. Instr. and Meth. A 315 (1992) 299;

S. Bianco et al., Nucl. Instr. and Meth. A 315 (1992) 322;

M. Bertino et al., Nucl. Instr. and Meth. A 315 (1992) 327;

B. Bencheikh et al., Nucl. Instr. and Meth. A 315 (1992) 354;

D. Acosta et al., Nucl. Instr. and Meth. A 316 (1992) 184;

D. Acosta et al., Nucl. Instr. and Meth. A 320 (1992) 128;

P. Mélèse et al., Nucl. Instr. and Meth. A 322 (1992) 189;

F. Takasaki et al., Nucl. Instr. and Meth. A 322 (1992) 211;

A. Martinis, INFN-AE-92-26, Nov. 1992;

M. Livan, RD1 - Scintillating Fibre Calorimetry, Proc. 3rd Int. Conf. on Calorimetry in High Energy Physics, Sept 29-Oct 2, 1992, Corpus Christi, Texas, p. 110; also CERN-PPE-93-22, Feb. 1993;

A. Asmone et al., ibid., p. 649; and Nucl. Instr. and Meth. A 326 (1993) 477;

E.C. Dukes et al., ibid., p. 655 ;

A. Ereditato et al., ibid., p. 677 ;

B. Worstell et al., ibid., p. 92;

S. Bianco et al., ibid., p. 791; 
D. Brown et al., Copper-Scintillating Fiber Hadron Calorimeter Tower Prototypes, presented at the 1992 IEEE Nuclear Science Symp., October 25-31, 1992, Orlando, Florida, Conf. Record, p. 274;

A. Martinis, INFN-AE-92-26 (1992);

J. Badier et al., CERN-PPE/93-20 (1993);

J. Badier et al., CERN-PPE/93-21 (1993);

B. Babusci et al., LNF-93/018 (1993);

S. Dagoret et al., Recent Development in Sci Fiber Calorimetry in the Framework of H1 Upgrade of Backward Calorimeter, presented at the 4th Int. Conf. on Calorimetry in High Energy Physics, Isola d'Elba, Italy, September 19-25, 1993;

U. Goerlach et al., A Lead Scintillating Fiber Calorimeter for the Upgraded H1 Detector, ibid;

$\mathrm{S}$. Miscetti et al., Performance of a Pb-Scintillating Fibre Calorimeter Prototype for the KLOE Experiment, ibid;

G. Anzivino et al., Projective Lead and Scintillating Fibre Calorımeter Test Beam Results, ibid;

A. Rimoldi et al., New Results on Scintillating Fibre Calorimetry, ibid;

B. Saitta et al., The High Resolution Calorimeter for the CHORUS Experiment, ibid.

[2] H. Fessler et al., Nucl. Instr. and Meth. A 240 (1985) 284; L. Labarga and E. Ros, Nucl. Instr. and Meth. A 249 (1986) 228;

B. Loehr et al., Nucl. Instr. and Meth. A 254 (1987) 26; G.S. Atoyan et al., Nucl. Instr. and Meth. A 320 (1992) 144;

R. Wigmans, Proc. 3rd Int. Conf. on Calorimetry in High Energy Physics, Corpus Christ1, Texas, Sept 29-Oct 2, 1992, p. 637 ;

S. White et al., ibid., p. 663;

A. Benvenuti et al., IEEE Trans. Nucl. Sci. NS-40 (1993) 537;

R. Tanaka et al., Shashlik Calorimeter for CMS, presented at the 4th Int. Conf. on Calorimetry in High Energy Physics, Isola d'Elba, Italy, September 19-25, 1993;

S. White et al., The PHENIX Calorimeter at RHIC, ibid.

[3] M.G. Albrow et al., Nucl. Instr. and Meth. A 256 (1987) 23 ;

J. Simon-Gillo et al., Nucl. Instr. and Meth. A 309 (1991) 427 ;

J.E. Simon-Gillo, Ph. D. Thesis. UMI-92-17024 (1991);

P. de Barbaro et al., Nucl. Instr. and Meth. A 315 (1992) 317;

D. Fox et al., Nucl. Instr. and Meth. A 317 (1992) 474;

M. Albrow, The CDF Plug Calorimeter Upgrade and Beam Tests of Tile-Fiber Calorimeters, contribution to the 1992 Int. Conf. on Advanced Technology and Particle Physics, Como, Italy, June 1992;

M. Lindgren, Proc. 3rd Int. Conf. on Calorimetry in High Energy Physics, Sept 29-Oct 2, 1992, Corpus Christi, Texas, p. 61;

J Proudfoot, ibid., p. 77;

P. Cushman, ibid., p. 321;

D.G. Underwood, bid,, p. 593;

T. Camporesi et al., CERN-PPE-92-212 (1992);

J.P. Sullivan et al., Nucl. Instr. and Meth. A 324 (1993) 441;
G. Apollinari et al., Nucl. Instr. and Meth. A 324 (1993)

475;

G. Apollinari et al., IEEE Trans. Nucl. Sci. NS-40 (1993) 484;

D. Autiero, et al., Nucl. Instr. and Meth A 336 (1993) 510;

A. Byon-Wagner et al., Study of Hadronic Shower Development Using the Reconfigurable - Stack Calorimeter, presented at the 4th Int. Conf. on Calorimetry in High Energy Physics, Isola d'Elba, Italy, September 19-25, 1993;

L. Poggıolı et al., Preliminary Results of an Iron/ Scintillating Tiles Hadron Calorimeter, ibid.;

P. De Barbaro et al., Tile-Fiber R\&D Results and the Hadron Calorimeter Production, ibid.;

M. Mishina et al., New Plug of CDF, ibıd.;

G. Apollinari et al., CDF Plug Upgrade: The Shower Maximum Detector, ibid.

[3a] D.G. Underwood, Proc. 3rd Int. Conf. on Calorimetry in High Energy Physics, Sept 29-Oct 2, 1992, Corpus Christi, Texas, p. 673;

A.F. Buzulutskov et al., ibid., p. 787;

V.I. Kryshkin et al., prepront IHEP 92-95, Protvıno, Russia (1992), submitted to Nucl. Instr. and Meth.;

B.V. Chuiko et al., 92-104, Protvino, Russia (1992), submitted to Nucl. Instr. and Meth.

[4] V. Burkert et al., CEBAF CLAS-Note-92-008 (1992).

[5] Raytech Industries Inc., P.O. Box 6-T, Stafford Sprıngs, CT, USA.

[6] Metallized Engineering Inc., P.O. Box 3036, Waterbury, CT 06705, USA.

[7] Bicron Corporation, 12345 Kingsman Rd., Newberry, $\mathrm{OH}$ 44065, USA.

[8] Type 1073D Tyvek, DuPont Fibers, Chestnut Run Plaza. P.O. Box 80705, Wilmington, DE 19880, USA.

[9] A. Andresen et al., Nucl. Instr. and Meth. A 309 (1991) 101.

[10] V. Hagopian, Florida State University, private communication.

[11] M. Kusner, Bicron Corporation, private communication and Bicron brochure, 1992.

[12] M. Kuhlen et al., Nucl. Instr, and Meth. A 301 (1991) 223.

[13] The spectra were kindly provided to us by M. Kusner, Bicron.

[14] E. Hernandez et al., SDC note SDC-92-291 (1992).

[15] NE Technology Limited, Sighthill, Edinburgh, Scotland.

[16] V. Burkert, CEBAF, private communication.

[17] The acrylic scintillator samples were kindly provided to us by John Lee of Polycast Technology Co, Box 141, Stamford, CT 06904, USA.

[18] Pol. Hi. Tech., Carsol1, Italy.

[19] Kuraray International Corporation, New York, NY 10166, USA.

[20] Burle Electron Tubes, Lancaster, PA 17601, USA.

[21] Thorn EMI Electron Tubes Inc., Rockaway, NJ 07866, USA.

[22] The samples of this PMT were kindly provided to us by Dr. E. Kistenev from Brookhaven National Laboratory.

[23] A. Weisenberger et al., Nucl. Instr. and Meth. A 327 (1993) 500. 
[24] A. Weisenberger et al., IEEE Trans. Nucl. Sci. NS-40 (1993) 455.

[25] M. Bertoldi et al., Radiat. Phys. Chem. 41 (1993) 413; K. Hara et al., Proc. 3rd Int. Conf. on Calorimetry in High Energy Physics, Sept 29-_oct 2, 1992, Corpus Christı, Texas, p. 598.

[26] Action Tool Services Inc., 2202 Mingee Street, Hampton, VA 23661, USA.

[27] The extruded scintillator samples were kindly provided to us by Dr. Kim Eglyan of the Yerevan Institute of Physics, Yerevan, Armenia.
[28] A. Byon-Wagner, Fermilab, private communication and ICFA Instrumentation Bulletin, No. 9 (April 1993) p. 23.

[29] S. Margulies, University of Chicago, private communication.

[30] K.F. Johnson, Florida State University, private communication.

[31] C. Zorn et al., Low Dose Rate Tests of Plastic Scintillator Plates and Wavelength Shifting Fibers, Contribution to the IV Int. Conf. on Calorimetry in High Energy Physics, Elba, Italy, Sept. 19-25, 1993. 